\section{Ruthenium(II)-diimin-Komplexe: Photosensibilisatoren mit einzigartigen Eigenschaften}

\author{
Peter Belser*
}

Abstract. Some Ruthenium(II)-diimine complexes have remarkable properties, which allow them to act as photosensitizers for the conversion of 'photon energy' into other utilizable energy forms. Presently, detailed knowledge of many properties in the ground and excited states of a great number of such complexes is available. This knowledge gives the possibility to prepare new compounds with predetermined properties. This article describes the most important properties of this type of compounds with respect to their photosensitizing ability. A discussion of binuclear ruthenium(II)-diimine complexes leads into the field of 'supramolecular chemistry'. The topic concepts 'photonic devices' and 'molecular devices' give interesting outlooks into possible future applications.

\section{Einleitung}

Die Untersuchung von Ruthenium(II)diimin-Komplexen wurde in den letzten 20 Jahren intensiv betrieben. Eine grosse Zahl von Publikationen zeugen von dieser Aktivität [1] [2]. Das grosse Interesse an dieser Verbindungsklasse hat verschiedene Gründe. Die Standardverbindung $\left[\mathrm{Ru}(\mathrm{bpy})_{3}\right]^{2+}$ bildet ein ideales Untersuchungsobjekt zum Studium der unterschiedlichsten Eigenschaften im Grundwie auch im angeregten Zustand [3]. Nach der 'Energiekrise' 1973 setzte man in diesen Metall-Komplex einige Hoffnung. Der Einsatz als Photosensibilisator bei der Wasserzerlegungsreaktion durch Sonnenlicht sah anfänglich recht vielversprechend aus. Ein geschlossener Reaktionszyklus, welcher als Nettoreaktion die Zerlegung von $\mathrm{H}_{2} \mathrm{O}$ in $\mathrm{H}_{2}$ und $\mathrm{O}_{2}$ zur Folge hat, konnte trotz Teilerfolgen nicht gefunden werden. Schwierigkeiten, wie z.B. die schnelle Rückreaktion der getrennten Ladungen oder das Fehlen eines vernünftigen 'Elektronenreservoirs' - es werden 4 Elektronen bei der Reaktion $2 \mathrm{H}_{2} \mathrm{O} \rightarrow 2 \mathrm{H}_{2}+\mathrm{O}_{2}$ umgesetzt - , sind noch nicht gelöst. Der grosse Aufwand, der in die Erforschung dieser Substanzklasse gesteckt wurde, brachte aber doch eine Fülle von Erkenntnissen, weiche z.B. auch ein tieferes Verständnis der Prozesse nach der Lichtabsorption ergaben. Die Entwicklung der Synthese und der Untersuchung der Ru-

\footnotetext{
* Korrespondenz: Dr. P. Belser Institut für Anorganische Chemie der Universität Freiburg i. Ü. Route du Musée, Pérolles $\mathrm{CH}-1700$ Fribours
}

thenium(II)-diimin-Komplexe kann sowohl zeitlich, als auch von der Komplexität der Verbindungen her betrachtet, in drei Abschnitte eingeteilt werden.

I) Mononukleare Komplexe. In dieser Entwicklungsstufe, welche heute als abgeschlossen gelten kann, wurden Ru-Verbindungen hergestellt, welche Diimin-Liganden in mannigfaltiger Variation enthalten. Besonders erfolgreich waren die Untersuchungen an 'Serien' $\left[\mathrm{Ru}(\mathrm{bpy})_{3-n}(\mathrm{~L})_{n}\right]^{2+}$ mit $n=0,1,2,3$ und $\mathrm{L}=$ Diimin-Ligand. Diese schrittweise Änderung der Ligand-Sphäre ermöglicht eine Aussage über die Tendenz der Veränderungen der Eigenschaften von der Standardverbindung $\left[\operatorname{Ru}(\mathrm{bpy})_{3}\right]^{2+}$ hin bis zum Komplex $\left[\mathrm{Ru}(\mathrm{L})_{3}\right]^{2+}$. Eine weitere Variation der Ligand-Sphäre bezüglich der Unterschiedlichkeit der Liganden $-\left[\mathrm{Ru}(\mathrm{L})_{3}\right]^{2+},\left[\mathrm{Ru}(\mathrm{L})_{x}\left(\mathrm{~L}^{\prime}\right)_{y}\right]^{2+}$ und $\left[\mathrm{Ru}(\mathrm{L})\left(\mathrm{L}^{\prime}\right)\right.$ $\left.\left(\mathrm{L}^{\prime \prime}\right)\right]^{2+}-$ ist in der Originalliteratur eingehend beschrieben [4]. Dank der grossen $\mathrm{Zahl}$ von Ru-Komplexen, welche bis heute dargestellt wurde, können massgeschneiderte Verbindungen aufgebaut werden, welche bestimmte und zum voraus festgelegte Eigenschaften aufweisen.

II) Polynukleare Komplexe und Käfig$V^{\prime}$ erbindungen. Diese beiden Zweige der Ru-Chemie, welche zur Zeit intensiv bearbeitet werden, beinhalten anspruchsvolle Synthese- und Trennprobleme. Es besteht bei den polynuklearen Komplexen die Möglichkeit, sowohl die Metalle als auch die Liganden und insbesondere auch die Brückenliganden zu variieren und deren Einfluss auf das gesamte Molekül zu studieren. Die Käfig-Verbindungen bilden eine getrennte Klasse von Komplexen, wel-

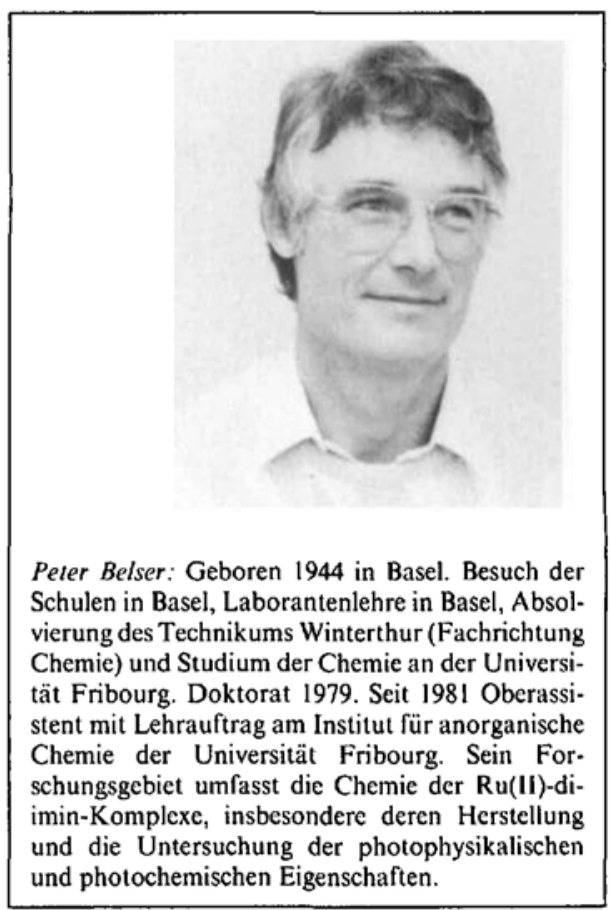

che zwecks Erhöhung der photochemischen Stabilität hergestellt werden.

III) 'Supramolekulare' Komplexe. Wir stehen heute an der Schwelle der Erforschung dieses faszinierenden Gebietes. Umfassende Kenntnisse der Herstellung und der Eigenschaften von mono- und polynuklearen Ru-Komplexen sind notwendig, um supramolekulare Verbindungen aufzubauen. Diese Verbindungen sollten in der Lage sein, verschiedene Funktionen gleichzeitig oder in der richtigen Zeitsequenz zu vollziehen, um in der Art eines molekularen Prozessors ("molecular device') zu arbeiten und gleichzeitig eine umfassendere, komplexere Funktion auszuführen.

Der vorliegende Artikel beschreibt in den ersten Kapiteln die wichtigsten Eigenschaften der mononuklearen Ruthenium(II)-diimin-Komplexe. Weitere Abschnitte sind den polynuklearen, den Käfig-Verbindungen und den 'supramolekularen' Komplexen gewidmet.

\section{Synthese der Komplexe}

\subsection{Wahl des Ligandensystems}

Allen $\mathrm{Ru}(\mathrm{II})$-diimin-Verbindungen ist ein Strukturelement gemeinsam. Es ist dies der 5gliedrige, cyclische Chromophor (Fig. 1), der sowohl das Zentralatom wie auch die Diimin-Funktion mit einschliesst.

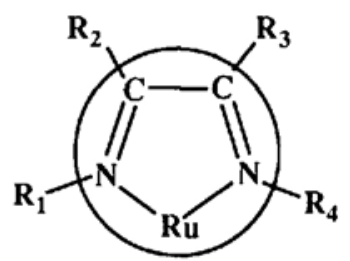

Fig. 1. Cyclischer, 5gliedriger Ru(II)-Diimin-Chromophor mit den Ligand-Fragmenten $R_{1}-R_{4}$ 


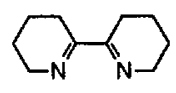

$1=$ H8-bpy

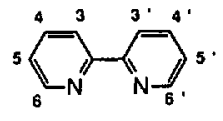

$\mathbf{2}=$ bpy

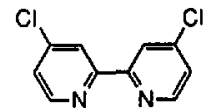

$3=4,4^{\prime}-d$ Cl-bpy

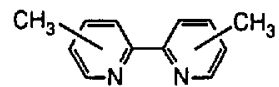

$\frac{4}{5}=3,3^{\prime}-d \mathrm{~m}-\mathrm{bpy}$

$\underline{6}=5,5^{\prime} \cdot \mathrm{dm}-\mathrm{bpy}$

$z=6,6^{\prime}-\mathrm{dm}-\mathrm{bpy}$

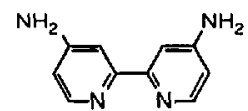

$8=4,4-d a-b p y$

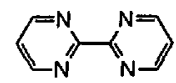

$\underline{9}=$ bipym

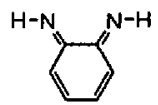

$10=$ opdi $\underline{5}=4,4^{\prime}-d \mathrm{~m} \cdot \mathrm{bpy}$

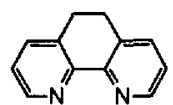

$11=H 2 \cdot$ phen

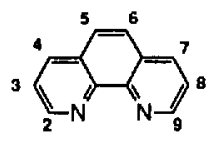

12 = phen

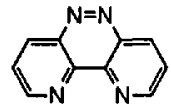

13 = taphen

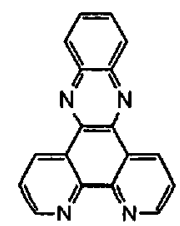

$14 \cdot \mathrm{dppz}$

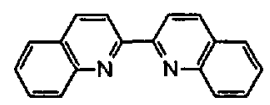

$\underline{15}=\mathrm{biq}$

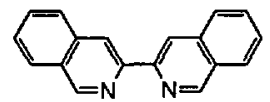

$\underline{16}=\mathrm{i}$-bia

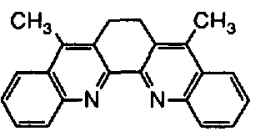

$17=\mathrm{DMCH}$
Dieses Strukturelement tritt im Komplex $\left[\mathrm{Ru}(\mathbf{1})_{3}\right]^{2+}$ auf. Dieser Chelat-Ring ist verantwortlich für die intensive Absorptionsbande, welche an allen Ru(II)-diiminKomplexen beobachtet wird. Er kann deshalb als 'Elementar-Chromophor' bezeichnet werden. In der Tat zeigt $\left[\mathrm{Ru}(1)_{3}\right]^{2+}$ dieselbe charakteristische MLCT-Bande [5] (metal-to-ligand charge transfer) wie die entsprechende Standardverbindung $\left[\operatorname{Ru}(\text { bpy })_{3}\right]^{2+}\left(=\left[\operatorname{Ru}(2)_{3}\right]^{2+}\right)$.

Um die Vielfalt der bis heute hergestellten $\mathrm{Ru}$ (II)-diimin-Komplexe $\mathrm{zu}$ ordnen, können sie grob nach folgenden Kriterien eingeteilt werden:

Grundsystem: Diimin-Ligand

2 ( = bpy) oder 12 ( = phen):

Einführung und Variation von Substituenten in den Positionen 3-6 resp. 3'-6' von 2 und $2-9$ von 12.

Die Substituenten üben einen induktiven und $z$. T. auch einen mesomeren Effekt auf die Elektronendichte am N-Atom des Liganden aus. Direkt korreliert mit der Elektronendichte am N-Atom ist auch die Bereitschaft zur Bildung einer ' $\pi$-Rückbindung' $R u \rightarrow N$. Diese beiden Effekte manifestieren sich deutlich in den photophysi-
Tabelle. Vergleich der langwelligsten MLCT-Übergänge $\left(\lambda_{\max }\right.$ in $\left.\mathrm{MeCN}\right)$ von Ru-Komplexen mit den entsprechenden Extinktionskoeffizienten $(\varepsilon)$

\begin{tabular}{lll}
\hline Komplex & $\lambda_{\max }[\mathrm{nm}]$ & \multicolumn{1}{l}{$\varepsilon$} \\
\hline$\left[\mathrm{Ru}\left(5,5^{\prime}-\mathrm{dm}-\mathrm{dppy}\right)_{3}\right]^{2+}$ & 440 & 14700 \\
{$\left[\mathrm{Ru}\left(6,6^{\prime}-\mathrm{dm}-\mathrm{bpy}\right)_{3}\right]^{2+}$} & 446 & 7440 \\
{$\left[\mathrm{Ru}\left(3,3^{\prime}-\mathrm{dm}-\mathrm{bpy}\right)_{3}\right]^{2+}$} & 456 & 11000 \\
{$\left[\mathrm{Ru}\left(4,4^{\prime}-\mathrm{dm}-\mathrm{bpy}\right)_{3}\right]^{2+}$} & 459 & 17000 \\
{$\left[\mathrm{Ru}\left(4,4^{\prime}-\mathrm{dCl} \text {-bpy }\right)_{1}\right]^{2+}$} & 462 & 17000 \\
{$\left[\mathrm{Ru}\left(4,4^{\prime}-\mathrm{da}-\mathrm{bpy}\right)_{3}\right]^{2+}$} & 504 & 10500 \\
{$\left[\mathrm{Ru}(\mathrm{bpy})_{3}\right]^{2+}$} & 452 & 14600 \\
\hline
\end{tabular}

kalischen Eigenschaften der Ru-Komplexe. Einerseits ist der Einfluss des gleichen Substituenten, z. B. auf die Lage des Absorptionsmaximums des MLCT-Übergangs unterschiedlich, je nach seiner Position am Pyridin-Ring (Me-Gruppe in Position 3, 4, 5 und 6 ; s. Tab. 1), andererseits wirkt sich die 'Art' des Substituenten bei gleicher Position ebenfalls auf die spektralen Eigenschaften aus. Beispiele solcher Substituenten sind die Me-Gruppe (5: $\lambda_{\max }$ $459 \mathrm{~nm}), \mathrm{Cl}\left(3: \lambda_{\max } 462 \mathrm{~nm}\right)$ oder die $\mathrm{NH}_{2}-$ Gruppe (8: $\lambda_{\max } 504 \mathrm{~nm}$ ). Weiterhin ist die Absorptionsintensität $(\varepsilon)$ abhängig von der Überlappung des Start- und des ZielOrbitals, welche am Elektronentransfer beteiligt sind. Bei sterisch bedingter Verlängerung der $\mathrm{Ru}-\mathrm{N}$-Bindung muss daher eine schwächere Absorption erwartet werden. Dies wird durch die $\varepsilon$-Werte in der Tab. 1 bestätigt. Die 6-Me-Gruppen von $\left[\mathrm{Ru}\left(6,6^{\prime}-\mathrm{dm}-\mathrm{bpy}\right)_{3}\right]^{2+}$ verlängern die $\mathrm{Ru}-\mathrm{N}-$ Bindung, was eine Verringerung des $\varepsilon$ Wertes zur Folge hat. Ein kleiner $\varepsilon$-Wert ist aber im Hinblick auf die Absorptionseigenschaft der Photosensibilisatoren nicht erwünscht.

Eine zusätzliche Variationsmöglichkeit besteht im Einbau weiterer Heteroatome in die beiden Liganden bpy (2) und phen (12). Beispiele solcher abgeänderter Ligandsysteme sind 9 und 13.

Sterische Hinderung (4), Nichtplanarität der beiden heterozyklischen Aromatenringe $(4,11$ und 17$)$ sowie konformativ verschiedene Anordnungen der Liganden bei der Komplexierung (11 und 17) sind Änderungen, welche ebenfalls Einfluss auf die photophysikalischen und photochemischen Eigenschaften der Komplexe haben [6].

Grundsystem: Verkleinertes $\pi$-System gegenüber 2 oder vergrössertes $\pi$-System gegenüber 12.

Die Veränderung der Grösse des $\pi$-Systems der Liganden ist eine weitere Möglichkeit, um die Eigenschaften der zu bildenden Photosensibilisatoren zu beeinflussen. Die Liganden 1 und 10 oder 14, 15, 16, und 17 können dieser Kategorie zugeordnet werden [5] [7].

\section{Spezialsysteme}

Im Übersichtsartikel von Juris et al. [3] ist eine grosse Zahl von weiteren LigandSystemen, welche sich nicht in das obige Schema einordnen lassen, aufgelistet.

\subsection{Synthese der \\ Ru(II)-diimin-Komplexe}

Die Herstellung der Ru-Komplexe ist, im Vergleich zu den $z$. T. aufwendigen $\mathrm{Li}$ gand-Systemen, eher unproblematisch [8]. Es bestehen unterschiedliche Synthesewege, die, je nach den gegebenen Randbedingungen, wie z. B. Loslichkeit, Reaktivität oder Möglichkeiten für Nebenreaktionen, eingeschlagen werden können oder eingeschlagen werden müssen.

a) Die Liganden und eine Ru-Verbindung (z. B. $\mathrm{RuCl}_{3} \cdot 3 \mathrm{H}_{2} \mathrm{O}$ oder $\mathrm{K}_{2} \mathrm{RuCl}_{5}$ ) werden in einem geeigneten Lösungsmittel wie EtOH, Ethylenglykol oder DMF erwärmt und anschliessend der Komplex aus dem Reaktionsgemisch isoliert. Nach diesem Verfahren lassen sich Komplexe vom Typus $\left[\mathrm{Ru}(\mathrm{L})_{2} \mathrm{Cl}_{2}\right]$ und $\left[\mathrm{Ru}(\mathrm{L})_{3}\right]^{2+}$ herstellen.

b) Thermischer oder photochemischer Abbau eines Komplexes nach folgender Reaktionsgleichung:

$$
\left[\mathrm{Ru}(\mathrm{L})_{3}\right] \mathrm{X}_{2} \underset{\left(\mathrm{X}=\mathrm{Cl}^{-} \text {oder } \mathrm{Br}^{-}\right)}{\longrightarrow}
$$

$$
\left[\mathrm{Ru}(\mathrm{L})_{2} \mathrm{X}_{2}\right]+\mathrm{L}
$$

Das Reaktionsprodukt wird anschliessend durch den Einbau eines Liganden L' nach der Methode $a$ in einen neuen Ru-Komplex umgewandelt.

c) Chemische Modifizierungen an komplexierten Liganden ergeben neue Verbindungen, die einerseits als solche auf ihre Eigenschaften hin untersucht werden können, andererseits sind solche umgewandelte $\mathrm{Ru}-$ Komplexe Quellen für neue $\mathrm{Li}$ ganden, wenn sie nach der Methode $b$ aus dem Komplex befreit und isoliert werden [9].

d) Einige spezielle Ru-Verbindungen lassen sich nur durch eine Templatreaktion am Metallzentrum herstellen. Als Beispiel kann die Synthese der Verbindung $[\mathrm{Ru}(22)]^{2+}$ (s. Schema) erwähnt werden [10].

Die Reindarstellung der Photosensibilisatoren ist im Falle der auf emittierende Verunreinigungen äusserst empfindlichen Messung der Emission von grundlegender Bedeutung. Umkristallisierung und verschiedene chromatographische Trennverfahren (Ionenaustausch, Flüssigkeits- und Gelchromatographie, und MPLC sowie präparative HPLC) sind die gebräuchlichsten Methoden, Verbindungen in gewünschter Qualität zu erhalten.

\section{Elektrochemie}

Mit Hilfe elektrochemischer Methoden wie der Cyclischen Voltammetrie (CV) können Informationen über die RedoxEigenschaften der Ru(II)-diimin-Komplexe gewonnen werden. Da die Redox-Eigenschaften der Photosensibilisatoren entscheidend die Wirksamkeit der Umwandlung von 'Photonenenergie' in andere, nutzbare chemische oder elektrische Ener- 


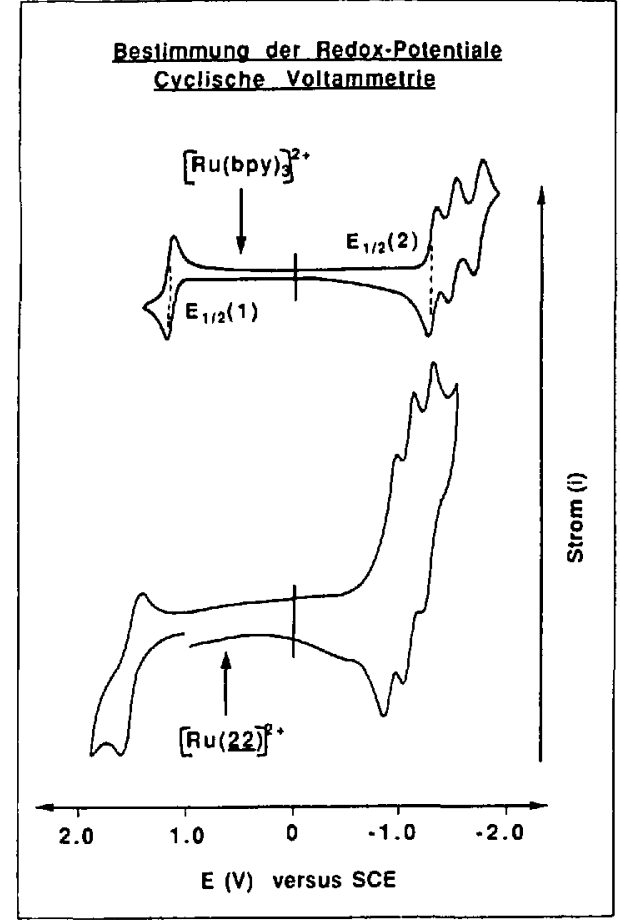

Fig. 2. Cyclische Voltammogramme der beiden Verbindungen $\left[R u(b p y)_{3}\right]^{2+}$ und $[R u(22)]^{2+}$. Im positiven Potentialbereich ist die Metalloxidation/reduktion und im negativen Potentialbereich sind die drei $\mathrm{Li}$ gandreduktionen/oxidationen zu beobachten.

gie beeinflusst, ist deren Bestimmung von eminenter Bedeutung. Je nach der Wahl der Liganden lassen sich die Redox-Potentiale der $\mathrm{Ru}$-Verbindungen einstellen ('tuning'). Zwei unterschiedliche Bereiche im $\mathrm{CV}$ können beschrieben werden. Im Bereich von $c a .+1,2 \mathrm{~V}$ ist der reversible $\mathrm{Re}$ dox-Vorgang $\mathrm{Ru}^{\mathrm{II} / \mathrm{III}}$ zu beobachten (s. Fig. $\left.2 ; E_{1,2}(1)\right)$. Zwischen $-1,3 \mathrm{~V}$ und $-2,0 \mathrm{~V}$ sind drei CV-Signale zu erkennen, welche je den 3 ligandzentrierten Reduktionsresp. Oxidationsprozessen

(Ligand) $+\mathrm{e}^{-} \rightleftarrows$ (Ligand)

entsprechen. Das erste dieser drei CV-Signale ist in Fig. 2 mit $E_{1,2}(2)$ bezeichnet. Es besteht eine gewisse Ähnlichkeit zu den, im nächsten Kapitel noch zu besprechenden spektroskopischen Übergängen, bei denen ebenfalls formal 'Redox-Vorgänge' stattfinden.

Fig. 3 zeigt den Zusammenhang zwischen den elektrochemischen und den spektroskopischen Prozessen. In $a$ ) wird entweder das $\mathrm{Ru}$ oxidiert oder reduziert $\left(\mathrm{Ru}{ }^{\mathrm{It} / \mathrm{ll}}\right)$ oder der Ligand reduziert oder oxidiert $\left(\mathrm{L} / \mathrm{L}^{-}\right)$. In $\left.b\right)$ ist ersichtlich, dass beim elektronischen Úbergang gleichzeitig das $\mathrm{Ru}$ oxidiert und der Ligand reduziert wird.

Beim Auftragen der $E_{1 / 2}$-Potentiale der Reduktion des ersten Liganden in den Metall-Komplexen gegenüber den energetischen Lagen der Absorptions- oder Emissionsbanden in den gleichen Komplexen werden lineare Zusammenhänge gefunden [11]. Dieser Befund erleichtert die Planung eines Ru-Komplexes, dessen Redoxpotentiale - des Metallzentrums und der Liganden - geeignet sind für den Einsatz der Verbindung als Photosensibilisator. Die spektroskopischen und die elektrochemischen Eigenschaften sind jedoch gekoppelt, d.h. sie können nicht unabhängig voneinander beim Aufbau eines Ru(II)-diimin-Komplexes durch die Wahl der Liganden eingestellt werden. Es muss darauf hingewiesen werden, dass der spektroskopisch angeregte Zustand eines Ru-Komplexes sowohl ein stärkeres Oxidations- als auch ein stärkeres Reduktionsmittel als der entsprechende Grundzustand darstellt.

Fig. 14 zeigt die elektrochemischen Da- ten der Verbindung $\left[\mathrm{Ru}(\mathrm{bpy})_{3}\right]^{2+}$ im Gesamtbild der photophysikalischen Eigenschaften der Rutheniumkomplexe.

\section{Photophysikalische Eigenschaften}

\subsection{Absorption}

Ru(II)-diimin-Komplexe zeigen im sichtbaren Spektralbereich charakteristische, intensive Absorptionsbanden ( $\varepsilon \approx 5000-25000$; s. Fig. 6 ). Die Erforschung des Ursprungs dieser Absorption ist Inhalt einer grossen Zahl von Publikationen [3] [12]. $\mathrm{Ru}(\mathrm{II})$ ist ein $\mathrm{d}^{6}$-System mit der elektronischen Konfiguration $\pi_{\mathrm{M}} e^{4} \pi_{\mathrm{M}} \mathrm{a}_{2}^{2}$ und weist in oktaedrischen Komplexen von der Art $\left[\mathrm{Ru}(L)_{3}\right]^{2+} D_{3}$ -

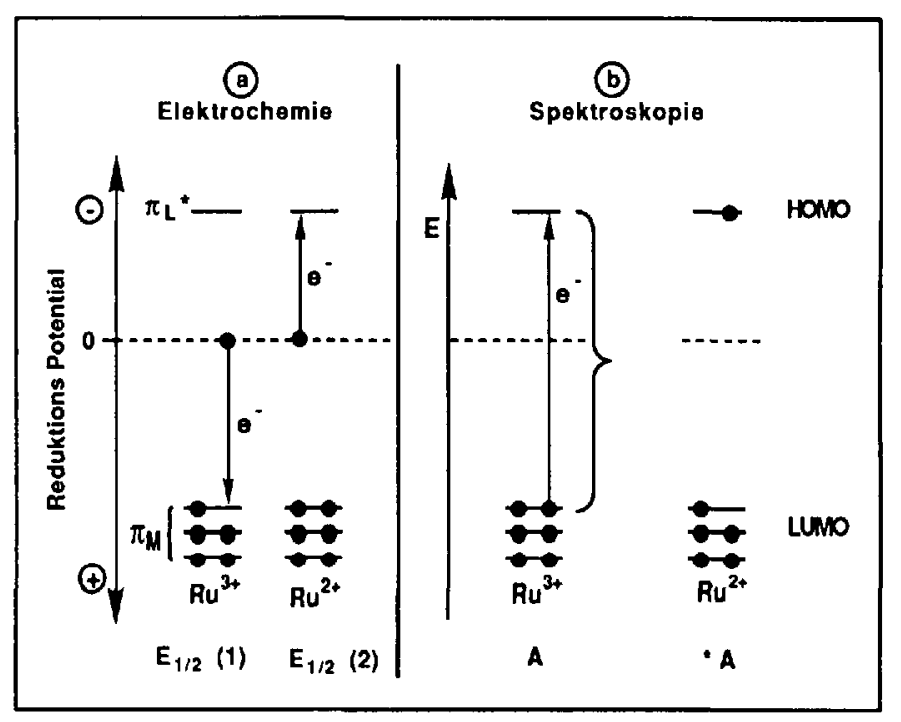

Fig. 3. Zusammenhang zwischen den Redox-Prozessen der Elektrochemie (a) und der spektroskopischen Anregung eines Elektrons (b)

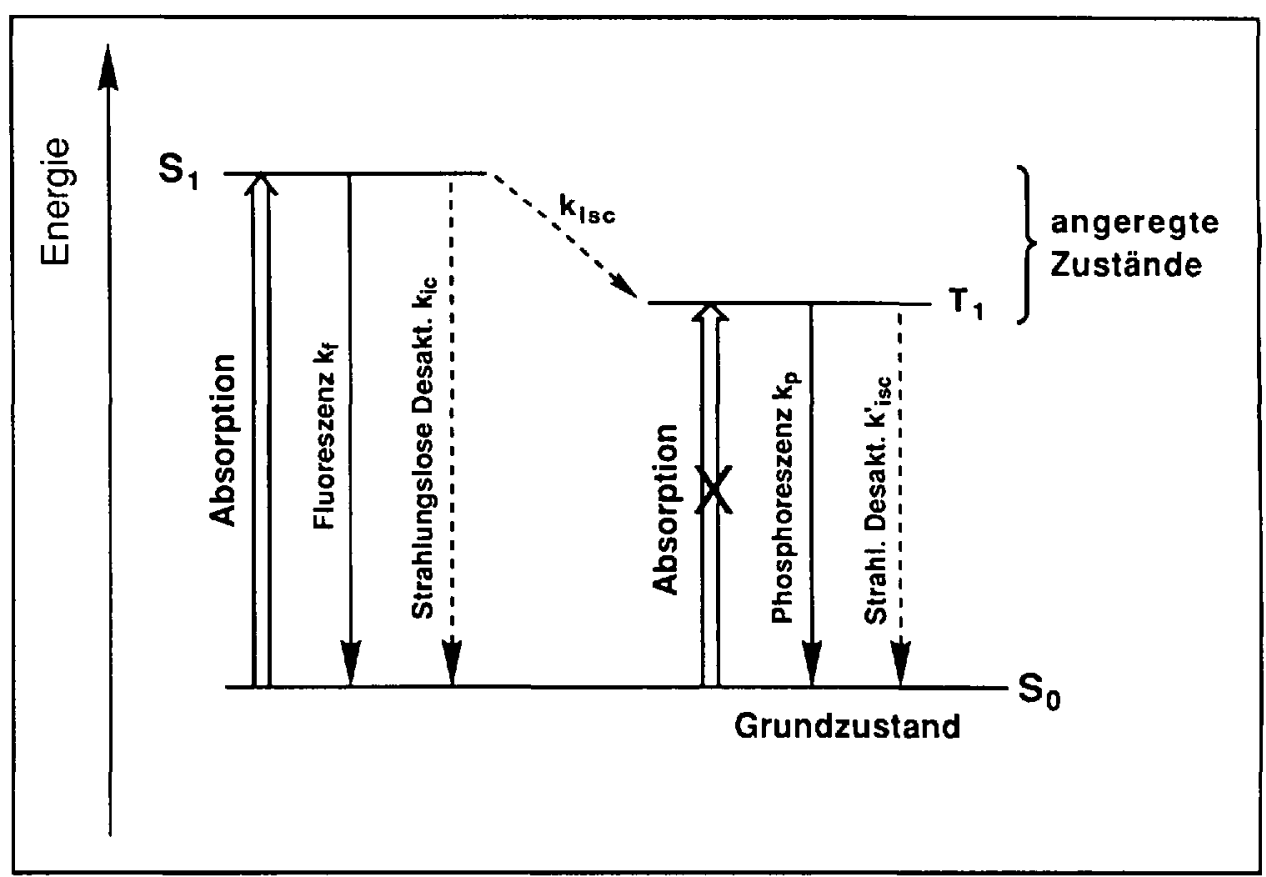

Fig. 4. Jablonsky-Schema der Komplexe $\left[R u(L)_{3}\right]^{2+}$ mit den iöglichen Desaktivierungsprozessen. Die direkte Absorption vom Grundzustand in den Triplett-Zustand $\left(T_{1}\right)$ ist spinverboten. 


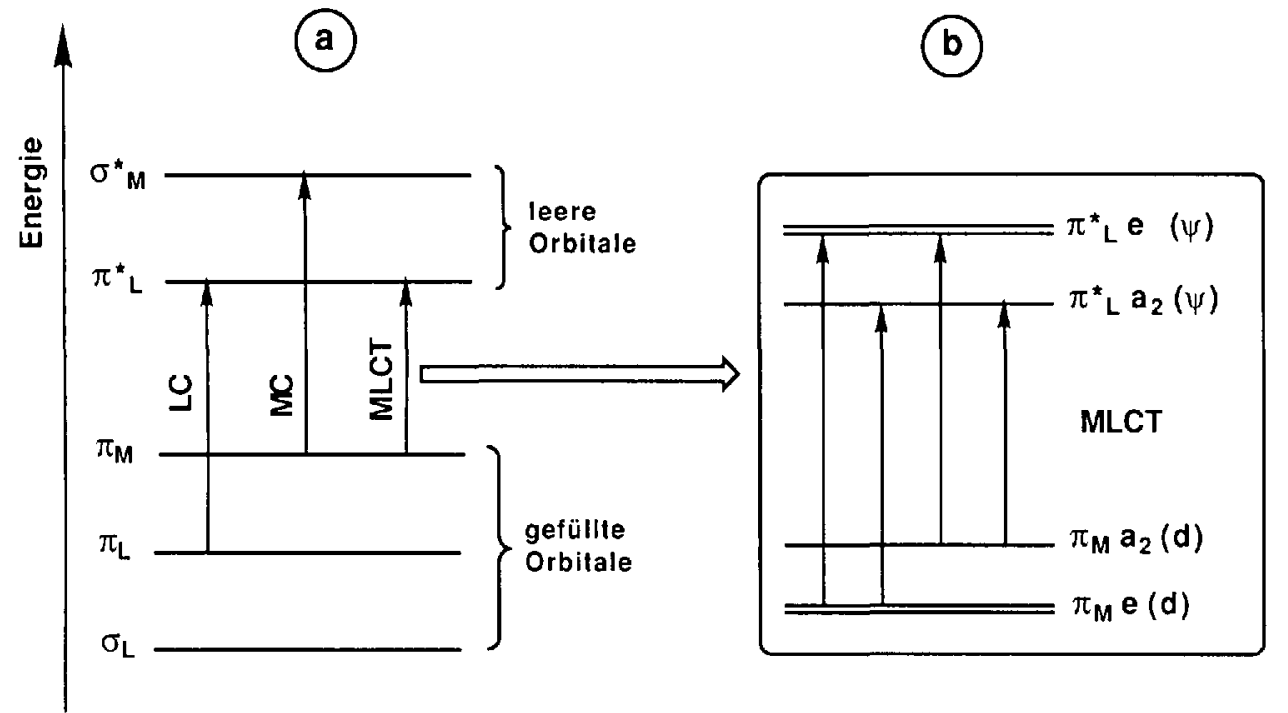

Fig. 5. MO-Schema der Komplexe $\left[R u(L)_{3}\right]^{2+}$ im HOMO/LUMO-Bereich. a) Drei unterschiedliche Übergänge LC, MC und MLCT. b) Detailliertere Darstellung der MLCTAnregung.

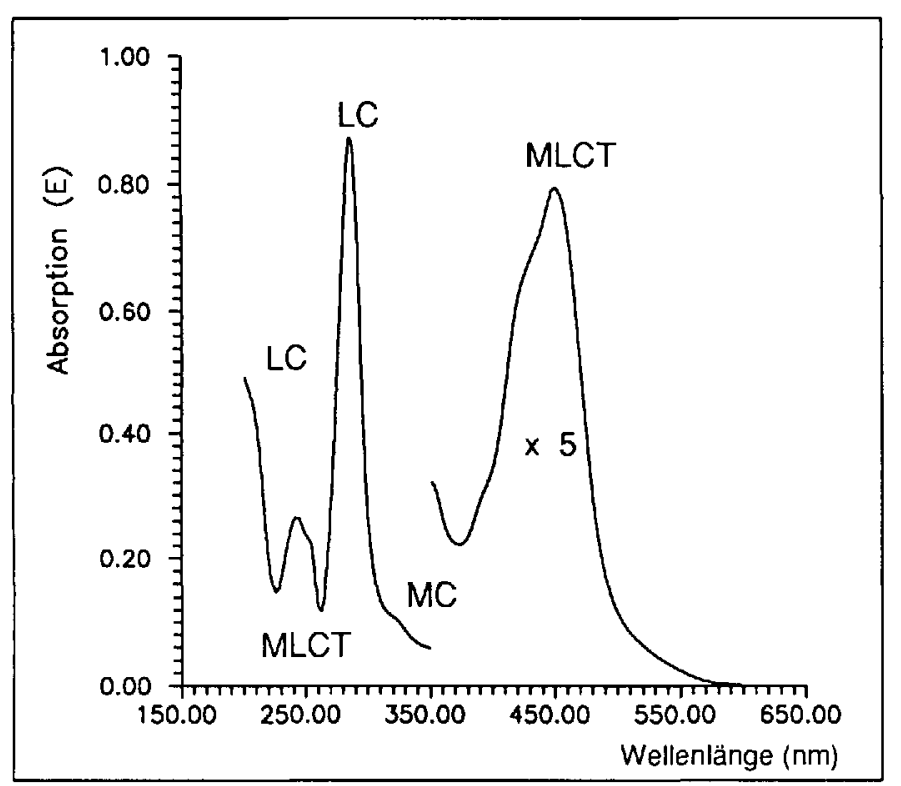

Fig. 6. Das Absorptionsspektrum von $\left[R u(b p y)_{3}\right]^{2+}$ im Bereich von $200-600 \mathrm{~nm}$. Es ist ersichtlich, dass sowohl MLCT-, MC- als auch LC-Übergänge im gleichen Molekül auftreten.

Symmetrie auf. In Fig. 4 ist ein vereinfachtes Jablonsky-Schema dargestellt. Die Absorption eines Photons promoviert den Komplex in einen angeregten SingulettZustand $\left(S_{1}\right)$. In einem effizienten Spinumkehrprozess (isc) wird ein Triplett-Zustand $\left(T_{1}\right)$ erreicht, der eine relativ lange Lebensdauer ( $\mu \mathrm{s}$ ) hat. Aus beiden Zuständen $S_{1}$ und $T_{1}$ kann die absorbierte Energie strahlungslos oder unter Aussendung eines Photons (Emission) abgegeben und so der Grundzustand wieder erreicht werden.

In der Fig. $5 a$ ist dieselbe Situation in einem vereinfachten MO-Schema gezeigt. Das Schema zeigt die möglichen Anregungen eines Elektrons in ein höher liegendes, leeres Orbital.

Der MC-Übergang entspricht einer Anregung eines Elektrons von einem $\pi_{M}$-Orbi- tal zu einem $\sigma_{\mathrm{M}}^{*}$-Orbital. Ligand-zentrierte Übergänge (LC) entsprechen dem Anregungsschema $\pi_{L} \rightarrow \pi_{L}^{*}$. Die MLCT-Absorption promoviert ein Elektron aus einem $\pi_{M}$-Orbital (metall-zentiert) in ein $\pi_{\mathrm{L}}{ }^{*}$.

Die detaillierter gezeichnete Fig. $5 b$ lässt erkennen, dass auch die MLCT-Bande aus mehreren, energetisch nahe beieinanderliegenden elektronischen Übergängen zusammengesetzt ist. Die Ligand-Feldstärke und die Redox-Eigenschaften der eingesetzten Liganden bestimmen die energetische Lage der drei Übergänge (MC, LC und MLCT). Es muss darauf hingewiesen werden, dass die Anregung, wie sie im MOSchema dargestellt wird, immer via einer Singulett-Anregung erfolgt. Erst nach dem Spinumkehrprozess wird der energetisch Orbital (ligand-zentriert) tiefer liegende Triplett-Zustand erreicht. In Fig. 6 ist das Absorptionsspektrum von $\left[\mathrm{Ru}(\mathrm{bpy})_{3}\right]^{2+}$ mit den zugeordneten Übergängen im Bereich von $200-600 \mathrm{~nm}$ dargestellt.

Bei der Anregung eines Elektrons aus dem Grundzustand in einem ${ }^{3} \mathrm{MC}-Z$ ustand ändert sich die Geometrie des Komplexes stark (s. Fig. 7). Die Geometrie des Komplexes wird hingegen bei der Anregung in den ${ }^{3} \mathrm{LC}$ - resp. ${ }^{3} \mathrm{MLCT}$-Zustand nur unwesentlich beeinflusst. Dieser Tatbestand ist entscheidend für das photophysikalische Verhalten der verschiedenen Ru-Komplexe.

Wenn der tiefste angeregte Zustand ${ }^{3} \mathrm{MC}$-Charakter hat, ist ein strahlungsloser Desaktivierungsprozess - Rückkehr in den Grundzustand resp. chemische Reaktion bevorzugt (s. Fig. 7a). Bei solchen Verbindungen wird keine Emission zu beobachten sein. $\left[\mathrm{Fe}(\mathrm{bpy})_{3}\right]^{2+}$ ist ein Beispiel eines solchen Metall-Komplexes mit einem LUMO-Orbital, welches MC-Charakter aufweist. Wenn hingegen der tiefste angeregte Zustand ein ${ }^{3} \mathrm{MLCT}$ - oder ${ }^{3} \mathrm{LC} \cdot \mathrm{Zu}$ stand ist, kann eine starke Emission beobachtet werden (s. Fig. 7 b). Diese Emission ist gleichzeitig ein Hinweis auf die Verfügbarkeit der im angeregten Zustand "gespeicherten' Energie, da der angeregte Zustand nicht durch strahlungslose Desaktivierungsprozesse oder intramolekulare chemische Reaktionen desaktiviert wird. Bei den meisten der bis heute hergestellten $\mathrm{Ru}(\mathrm{II})$-diimin-Komplexen ist der tiefste angeregte ${ }^{3} \mathrm{MLCT}-Z$ ustand verantwortlich für die starke Emission, die gemessen werden kann. Dies gilt insbesondere für die Standardverbindung $\left[\mathrm{Ru}(\mathrm{bpy})_{3}\right]^{2+}$. Die Verbindung $\left[R u(16)_{3}\right]^{2+}$, die eingehend untersucht worden ist [13], besitzt cinen ${ }^{3} \mathrm{LC}$ Zustand als tiefstes angeregtes Niveau.

Ein wichtiger Aspekt, der von praktischer Bedeutung ist, ist die Tatsache, dass die Vielfalt der Ru-Komplexe, welche heute zur Verfügung stehen, ${ }^{3}$ MLCT-Banden liefern, die das ganze sichtbare Spektrum abdecken. CT-Übergänge im sichtbaren Bereich von $375.680 \mathrm{~nm}$ werden gefunden [3]. Somit kann eine Mischung geeigneter Ru-Verbindungen einen wesentlich grösseren Anteil an Sonnenlicht absorbieren, als dies $\left[\mathrm{Ru}(\mathrm{bpy})_{3}\right]^{2+}$ als einzelne Komponente tun könnte. Anhand von Absorptionsspektren von Komplex-Serien kann der Trend der photophysikalischen Veränderungen, die durch Ligand-Modifizierungen bedingt sind, klar demonstriert werden (Fig. 8). Zum Beispiel sind in der Serie $\left[\mathrm{Ru}(\mathrm{bpy})_{3-n}(\mathrm{biq})_{n}\right]^{2+}$ zwei gut getrennte MLCT-Übergänge zu erkennen. Sie können in erster Näherung den Übergängen $\mathrm{Ru} \rightarrow$ bpy und $\mathrm{Ru} \rightarrow$ biq zugeordnet werden und sind nur noch durch die lokalen Symmetrie-Eigenschaften $\left(D_{3} ; C_{2} ; C_{2} ; D_{3}\right)$ der jeweiligen Komplexe beeinflusst [14].

\subsection{Emission}

Eine der bemerkenswertesten Eigenschaften der $\mathrm{Ru}(\mathrm{II})$-diimin-Verbindungen ist die vielfach beobachtete Emission von 


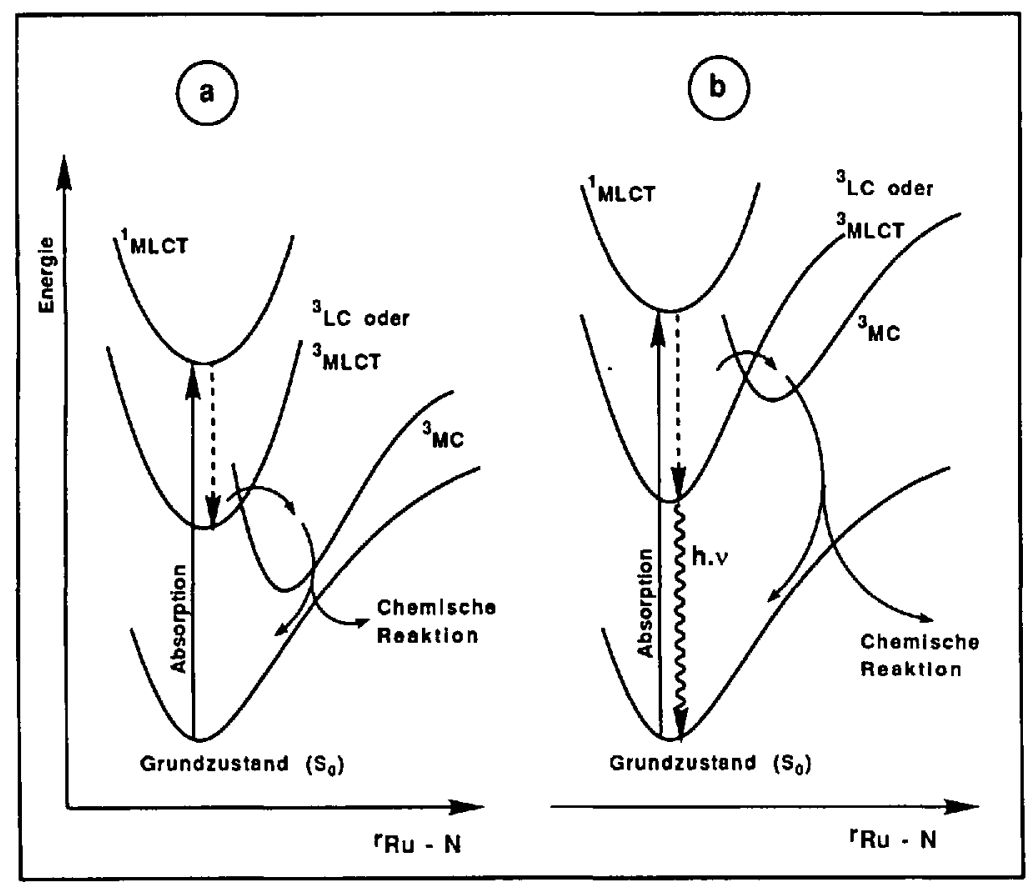

Fig. 7. Einfluss der energetischen Lage der ${ }^{3} M C$ - und $\operatorname{der}{ }^{3} M L C T$ (resp. ${ }^{3} L C$ )-Zustände auf das Emissionsverhalten bzw. die photochemische Reaktivität. a) Keine Emission; Thermische Desaktivierung oder photochemische Reaktion. b) Die Desaktivierung des Elektrons durch Emission wird vor der strahlungslosen Rückkehr des Elektrons via dem ${ }^{3} \mathrm{MC}-Z u s t a n d$ in den Grundzustand $\left(\mathrm{S}_{\mathrm{o}}\right.$ ) oder einer photochemischen Reaktion bevorzugt.

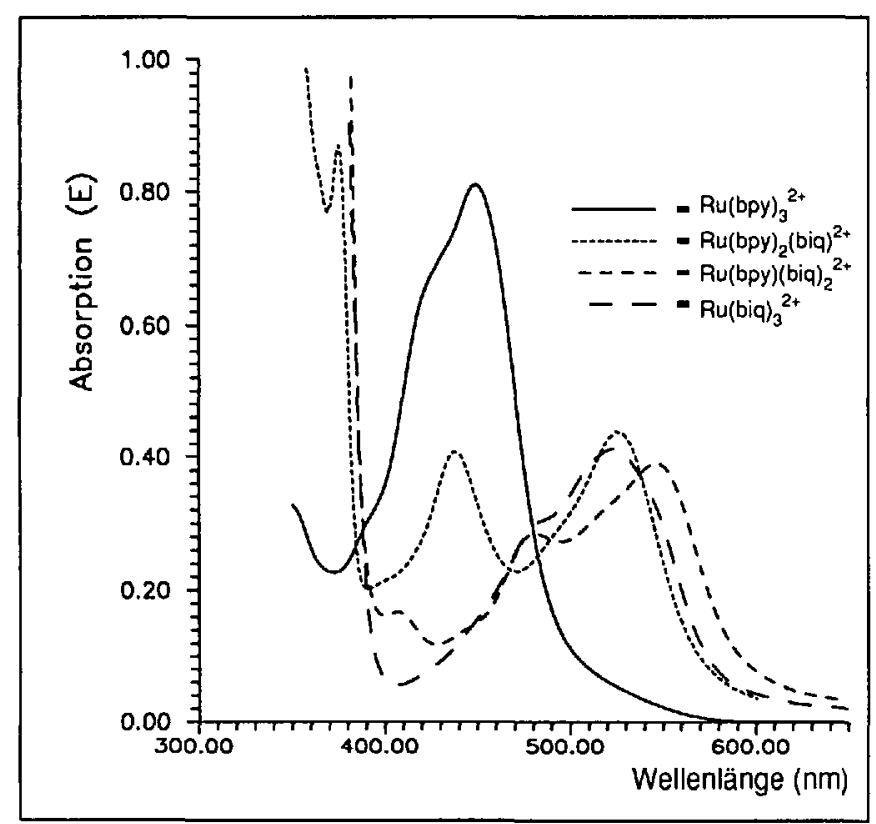

Fig. 8. Absorptionsspektren der Serie $\left[R u(b p y)_{3-\mathrm{n}}(\text { biq })_{\mathrm{n}}\right]^{2+}$

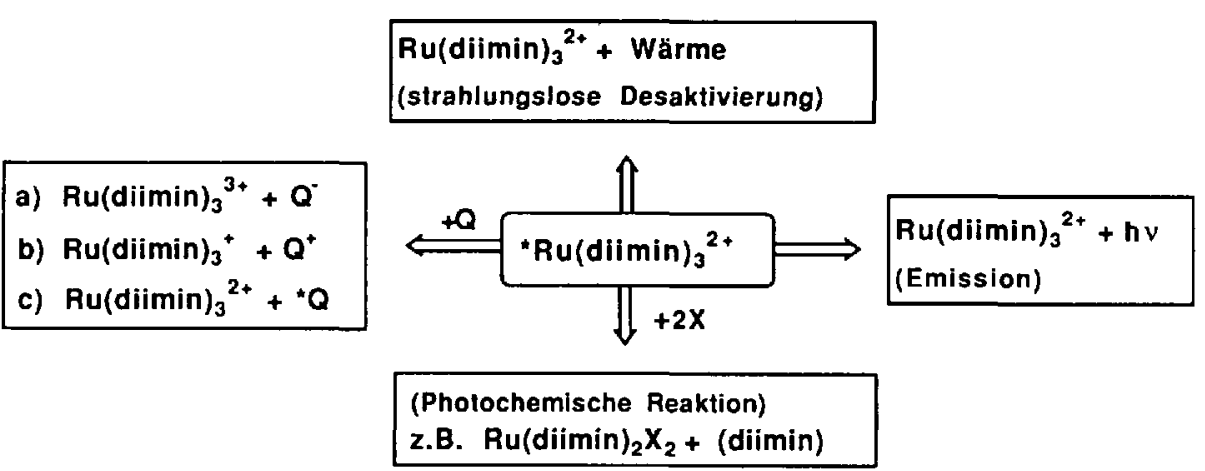

Fig. 9. Schematische Darstellung der Desaktivierungswege eines angeregten $\left[R u(L)_{3}\right]^{2+}$ Moleküls. $\mathrm{Q}=$ Quentscher. a) Oxidative Löschung der Emission. b) Reduktive Löschung der Emission. c) Energietransfer; $\mathrm{X}=$ z. B. $\mathrm{Cl}^{-}$. sichtbarem Licht. Die Emission erfolgt in der Regel aus dem tiefsten, elektronisch angeregten Zustand.

Die Emission lässt sich durch die Lebensdauer des angeregten Zustandes $(\tau)$ und der Quantenausbeute $(\phi)$ charakterisieren. $\phi$ entspricht dem Verhältnis der eingestrahlten, gegenüber den emittierten

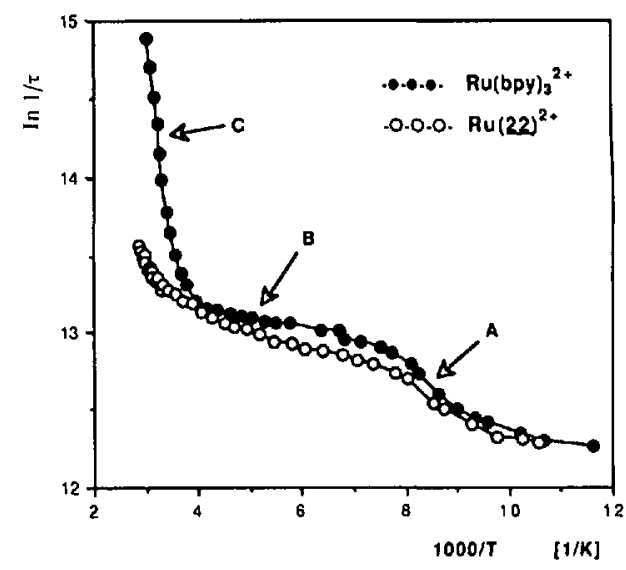

Fig. 10. Unterschiedliches Verhalten der Lebensdauer der Emission in Funktion der Temperatur der Standardverbindungen $\left[R u(b p y)_{3}\right]^{2+}$ und der Käfig-Verbindung $[R u(22)]^{2+}$. Die Emissionslebensdauer von $\left[\mathrm{Ru}(\mathrm{bpy})_{3}\right]^{2+}$ sinkt oberhalb $250 \mathrm{~K}$ drastisch ab [10c].

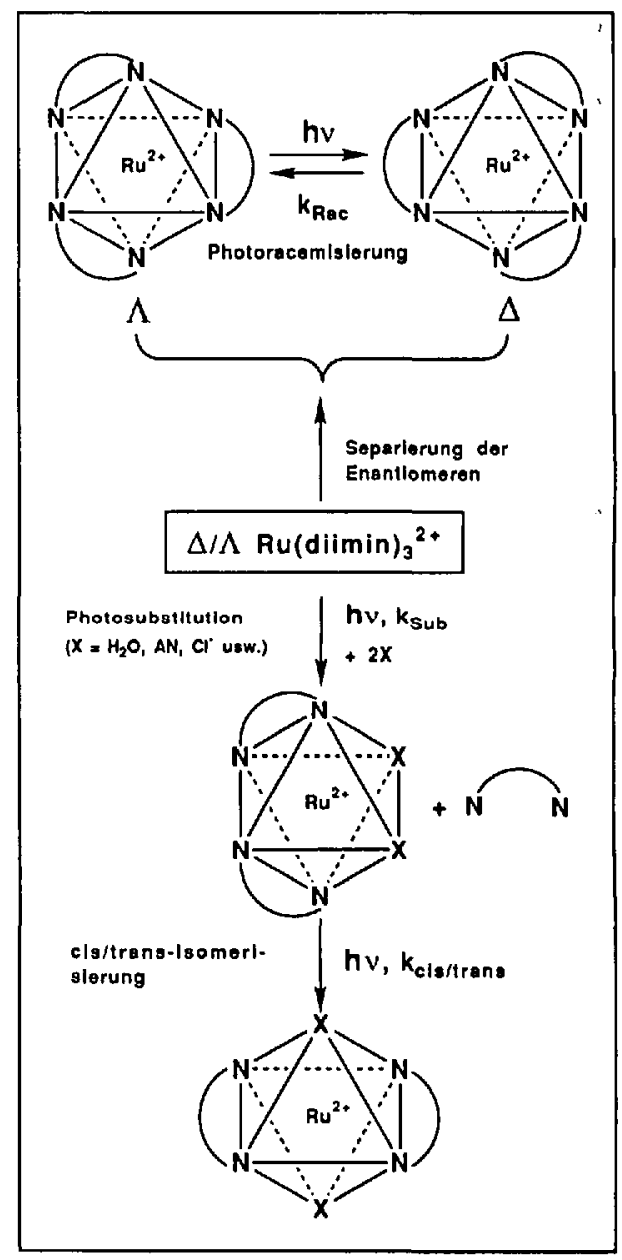

Fig. 11. Photoracemisierung, Photosubstitution und (cis/trans)-Isomerisierung mit den entsprechenden 'Geschwindigkeitskonstanten' $\mathrm{k}_{R a c}, \mathrm{k}_{S u b}$ und $\mathrm{k}_{\mathrm{cis} \text { itrans }}$ 
Quanten. Bei der Emission aus dem ${ }^{3}$ MLCT-Zustand von $\left[\mathrm{Ru}(\mathrm{bpy})_{3}\right]^{2+}$ bei Raumtemperatur beträgt $\tau=0,6 \mu$ s und $\phi=0,04$. Die Lebensdauer des angeregten Zustandes ist ebenfalls ein direkter Hinweis auf die Verfügbarkeit der 'gespeicherten' Energie. Mittels geeigneter Quencher kann die Energie via Elektronen- oder Energietransfer auf ein anderes Molekül übertragen werden (Fig. 9).

\subsection{Temperaturabhängigkeit der \\ Emissionslebensdaue}

Die Emissionslebensdauer $\tau$ der RuKomplexe ist stark temperaturabhängig [13] [15]. In Fig. 10 ist $\ln 1 / \tau$ gegenüber der reziproken Temperatur $\left(\mathrm{K}^{-1}\right)$ aufgetragen. Wir können drei verschiedene Bereiche unterscheiden. Im Gebiet $A$ schmilzt die gefrorene Matrix auf. Dies bedeutet eine Verminderung der Lebensdauer. Dieser Effekt kann mittels einer vermehrten vibronischen Desaktivierung des angeregten $\mathrm{Zu}$ standes erklärt werden. Der leichte Anstieg des Wertes $\ln 1 / \tau$ im Gebiet $B$ entspricht einer thermischen Besetzung $(\Delta E=90$ $\mathrm{cm}^{-1}$ ) eines angeregten Zustandes, welcher knapp über dem im Kap. 4.1 besprochenen ${ }^{3}$ MLCT-Zustand liegt und von gleicher elektronischer Natur ist. Der starke Anstieg im Gebiet $C$, welcher einer drastischen Verkürzung der Lebensdauer des angeregten Zustandes entspricht, ist auf eine thermische Besetzung des photoreaktiven ${ }^{3} \mathrm{MC}$-Zustandes ( $\mathrm{ca} .3000-5000 \mathrm{~cm}^{-1}$; s. Fig. $5 a$ ) zurückzuführen. Aus dem mathematischen Modell [3], welches den Kurvenzug der Temperaturabhängigkeit von $\tau$ simuliert, können die angegebenen Energiedifferenzen $\Delta E$ der thermisch erreichbaren Energieniveaus bestimmt werden. Im folgenden Kapitel wird eine weitere Möglichkeit der Bestimmung dieser $\Delta E$-Werte besprochen.

\section{Photochemische Eigenschaften}

Wie aus Fig. Sa und 9 ersichtlich ist, kann das angeregte Molekül, welches sich im ${ }^{3} \mathrm{MC}-$ Zustand befindet, in einem intramolekularen Prozess seine Energie abgeben (Photoracemisierung) oder in einem dissoziativen Reaktionsschritt (Photosubstitution) in einen neuen Komplex umgewandelt werden. In Fig. 11 sind die beiden Reaktionswege dargestellt.

\subsection{Photoracemisierung}

Alle Ru(II)-diimin-Komplexe fallen be der Synthese als racemisches Gemisch an. Mit geeigneten Methoden [16] lassen sich die Enantiomeren trennen und bilden so ideale Ausgangsverbindungen zur Untersuchung der photochemischen Reaktivität, insbesondere der Photoracemisierung. $\mathrm{Da}$ die Erreichung des photoreaktiven ${ }^{3} \mathrm{MC}$ Zustandes aus dem nicht reaktiven ${ }^{3}$ MLCT-Zustand thermisch entsprechend der Boltzmann-Theorie erfolgt, muss die

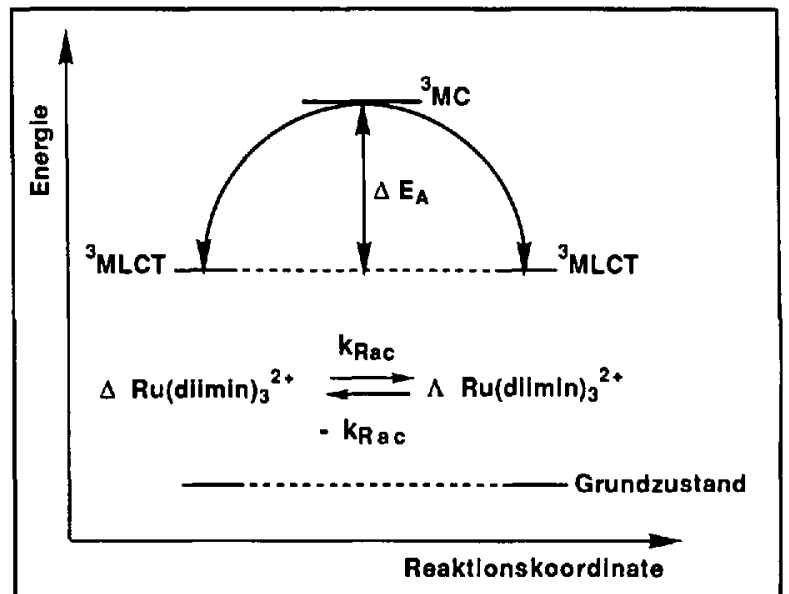

Fig. 12. Energetische Lage der Zustände eines einheitlich substituierten Ru(II)-DiiminKomplexes bezüglich einer Photoracemisierung

relative Racemisierungsgeschwindigkeit temperaturabhängig sein. In der Tat können bei der Messung der Geschwindigkeit der Racemisierung - Abnahme des Dreh-
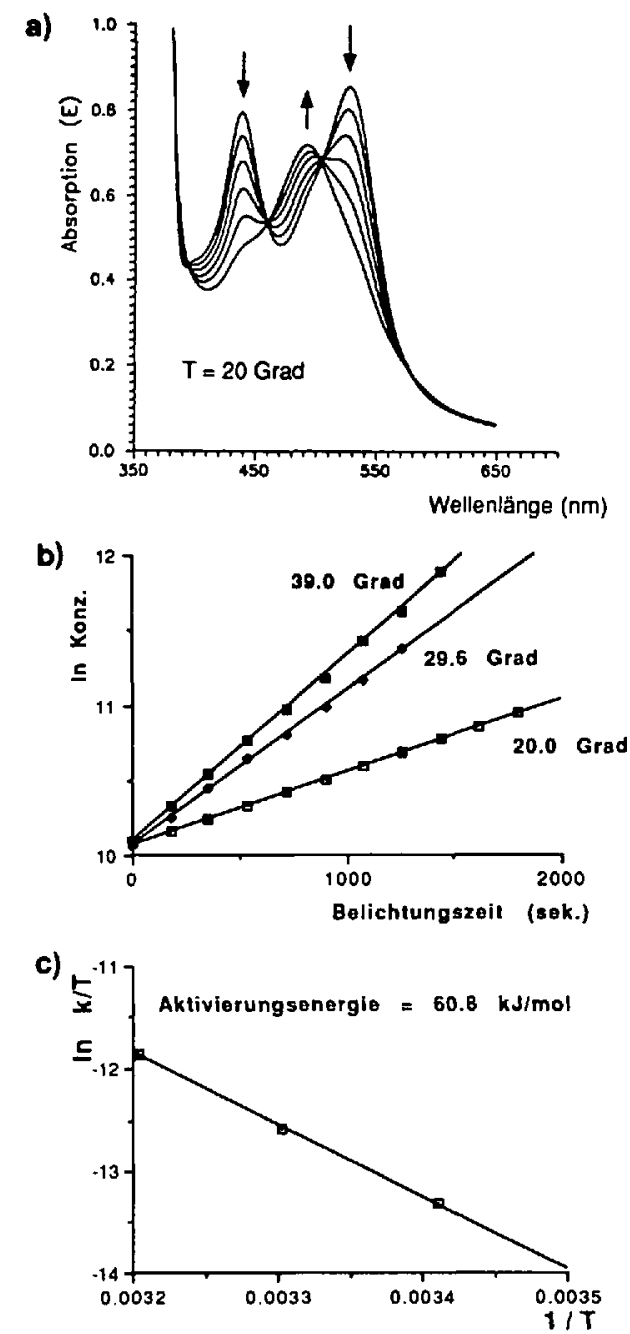

Fig. 13. Photosubstitution der Verbindung $\left[R u(b p y)_{2}(b i q)\right]^{2+}$. a) Absorptionsspektrum $=\mathrm{f}$ (Belichtungszeit). b) In Konz. $=\mathrm{f}$ (Belichtungszeit). Aus der Steigung kann die 'Geschwindigkeitskonstante' $k$ berechnet werden. c) $\ln k / T=\mathrm{f}(1 / T)$. Aus der Steigung dieser Funktion lässt sich die Aktivierungsenergie berechnen. wertes $\alpha$ oder des CD-Signals in Funktion der Bestrahlungsdauer - bei verschiedenen Temperaturen die Aktivierungsenergien, welche zum Erreichen des ${ }^{3} \mathrm{MC}$-Zustandes notwendig sind, nach Arrhenius berechnet werden [17]. Diese Werte stimmen bei der Annahme einer Reaktion pseudo-erster Ordnung gut mit den Werten überein, welche aus der Temperaturabhängigkeit der Emissionslebensdauer bestimmt worden sind. Zwei Argumente unterstreichen die Aussagekraft dieser Experimente. Zum einen ist der Energieinhalt der Ru-Komplexe vor und nach der Racemisierung identisch, d.h. die Aktivierungsenergie der Hin- und Rückreaktion ist dieselbe (s. Fig. 12). Diese Bedingung vereinfacht die Interpretation der 'kinetischen' Daten.

Andererseits können die Experimente mit Verbindungen durchgeführt werden, welche nur partiell mit einem der beiden optischen Isomeren angereichert sind. Wenn zusätzlich noch die 'reale Konzentration' des Anteils an optisch aktivem Komplex bekannt ist, können auch Aussagen über Entropie-Änderungen zwischen dem Grund- und dem Übergangszustand gemacht werden. Zudem kann im gleichen Experiment auch die Photosubstitution gemessen werden, wenn die beiden ' $\mathrm{Ge}$ schwindigkeitskonstanten' $k_{\mathrm{Rac}}$ und $k_{\mathrm{Sub}}$ von vergleichbarer Grössenordnung sind.

\subsection{Photosubstitution}

Das Beobachtbare bei der Messung der Photosubstitution ist die Änderung des Absorptionsspektrums in Funktion der Bestrahlungsdauer. Es wird jeweils in die langwelligste MLCT-Bande eingestrahlt. In Fig. 13 ist ein Beispiel einer Mess-Serie mit der entsprechenden graphischen Bestimmung von ' $k_{\text {sub }}$ ' der Verbindung $[\text { Ru(bpy })_{2}$ (biq) $]^{2+}$ gegeben. Die Reaktionsgleichung lautet:

$$
\left[\mathrm{Ru}(\mathrm{bpy})_{2}(\mathrm{biq})\right]^{2+} \frac{h v}{\begin{array}{c}
\text { Aceton } / \mathrm{H}_{2} \mathrm{O} \\
\mathrm{pH}=1,5
\end{array}}
$$

$\left[\mathrm{Ru}(\mathrm{bpy})_{2}\left(\mathrm{H}_{2} \mathrm{O}\right)\right]^{2+}+\mathrm{biq}$ 


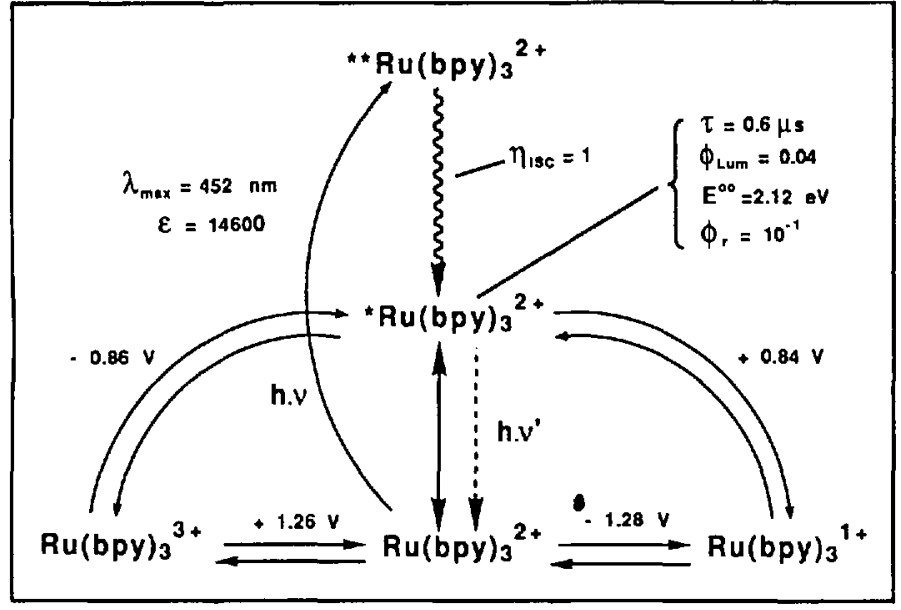

Fig. 14. Daten der Energie- und Elektronentransferprozesse der Verbindung $\left[R u(b p y)_{3}\right]^{2+}$. $\left[{ }^{* *} \mathrm{Ru}(\mathrm{bpy})_{3}\right]^{2+}$ entspricht dem $\mathrm{S}_{1}$ - und ${ }^{*} \mathrm{Ru}(\mathrm{bpy})_{3}{ }^{2+}$ dem tiefsten spinverbotenen ${ }^{3} \mathrm{MLCT}$ Zustand $\left(\mathrm{T}_{1}\right)$.

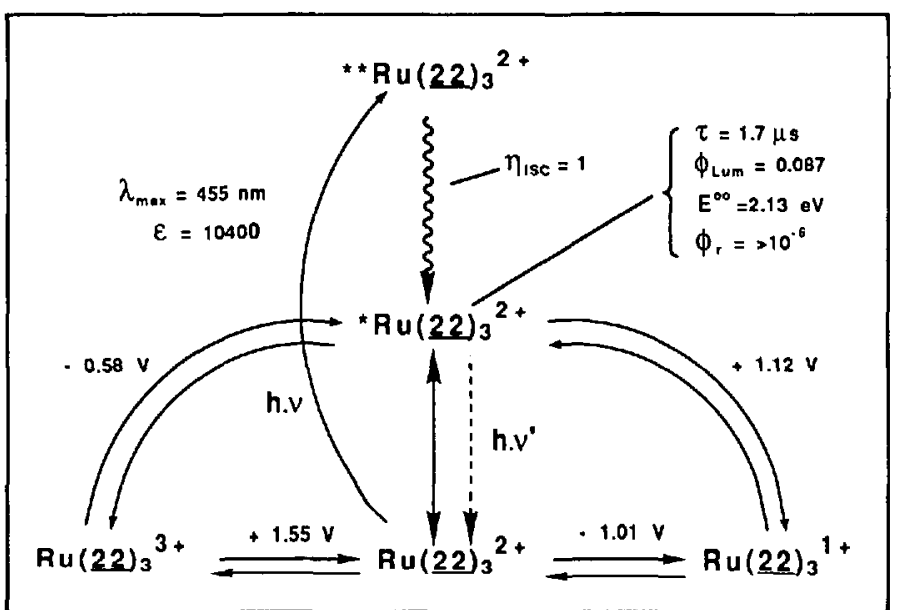

Fig. 15. Daten der Energie- und Elektronentransferprozesse der Verbindung $[R u(22)]^{2+}$ (s.a. Fig. 14)

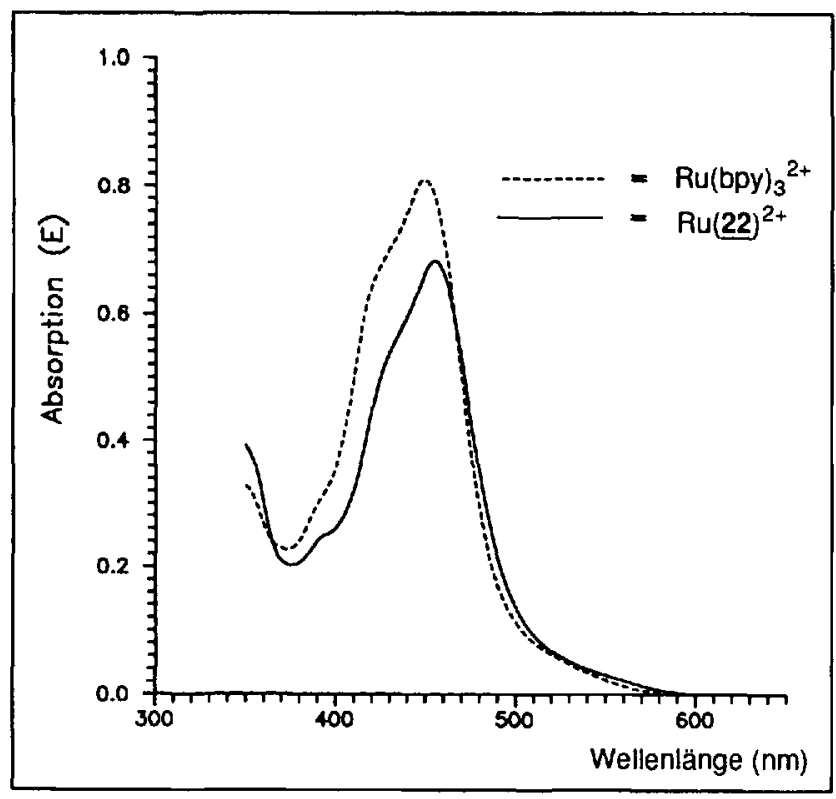

Fig. 16. Vergleich der Absorptionsspektren der Verbindungen $\left[R u(b p y)_{3}\right]^{2+}$ und $[R u(\mathbf{2 2})]^{2+}$
Es wird der sterisch stark gehinderte biqLigand (15), welcher schon im Grundzustand eine, gegenüber dem bpy-Liganden (2) verlängerte $\mathrm{Ru}-\mathrm{N}$-Bindung aufweist, ausgestossen.

Die bei verschiedenen Temperaturen gemessenen relativen Geschwindigkeitskonstanten der Photosubstitution ergeben nahezu dieselben Aktivierungsenergien wie bei der Photoracemisierung. Es wird also in beiden Fällen derselbe reaktive Übergangszustand $\left({ }^{3} \mathrm{MC}\right)$ erreicht [18]. Neuere Messungen haben jedoch gezeigt, dass es auch Ru-Verbindungen gibt, deren photochemische Prozesse nach einem komplexeren Reaktionsschema ablaufen [19].

Die (cis/trans)-Isomerisierung, die in Fig. 11 als weitere photochemische Reaktion dargestellt wird, eröffnet einen zusätzlichen Synthese-Weg zur Herstellung des thermodynamisch ungünstigeren trans Komplexes [20]. Diese Reaktionsabfolge ist nur bei sterisch anspruchslosen Liganden wie z. B. bpy (2) zu beobachten und muss als mögliche Nebenreaktion in die kinetische Beschreibung der photochemischen Reaktion miteinbezogen werden.

Zusammenfassend sind die Grundzustandseigenschaften und die Eigenschaften des angeregten Zustandes der Verbindung $\left[\mathrm{Ru}(\mathrm{bpy})_{3}\right]^{2+}$ in Fig. 14 aufgeführt.

\section{Käfig-Komplexe}

Wie am Anfang dieses Artikels erwähnt, ist die Synthese von Ru(II)-diimin-KäfigKomplexen erst vor kurzem gelungen [10]. Der Grund, der uns in dieses neue, präparativ anspruchsvolle Gebiet führte, lag in gewissen Nachteilen, welche die StandardVerbindung $\left[\mathrm{Ru}(\mathrm{bpy})_{3}\right]^{2+}$ in gelöstem $\mathrm{Zu}$ stand aufweist. Es wird eine schnelle, strahlungslose Desaktivierung des tiefsten angeregten Zustandes beobachtet, welche die Effizienz des Energie- resp. Elektronentransfers indirekt beeinflusst. Je schneller die Desaktivierung stattfindet, desto ungünstiger ist die Ausnutzung der im angeregten Zustand gespeicherten Energie. Im weiteren ist die photochemische Reaktivität zwar bei Syntheseproblemen anwendbar, für den Einsatz eines Photosensibilisators aber unerwünscht. Bestrebungen, diese Nachteile durch gezielte Ligand-Variationen zu beheben, wurde durch die Einführung neuer Nachteile verunmöglicht. So kann eine grössere photochemische Stabilität durch eine Erhöhung der EnergieBarriere zwischen dem ${ }^{3}$ MLCT- und dem thermisch erreichbaren ${ }^{3} \mathrm{MC}$-Zustand gewonnen werden, weil dadurch bei gleicher Temperatur die Population des reaktiven ${ }^{3} \mathrm{MC}$-Zustandes sinkt. Bisher gelang eine Vergrösserung der Energie-Barriere nur durch ein Absenken des ${ }^{3}$ MLCT-Zustandes, da eine Vergrösserung der LigandFeldstärke bei gleichbleibender Energie der $\pi^{*}$-Orbitale nur sehr schwer realisierbar ist. Dieses Absenken bringt aber eine Verkürzung der Lebensdauer des angeregten Zustandes mit sich, da die strahlungs- 


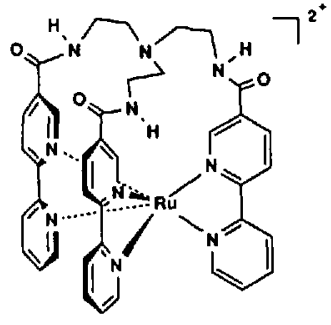

$\operatorname{Ru}(18)^{2+}$

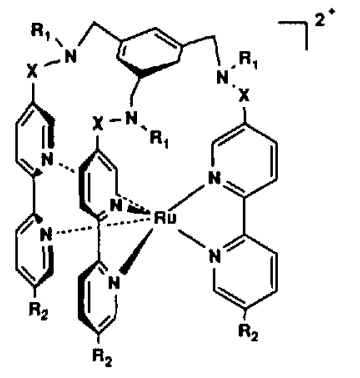

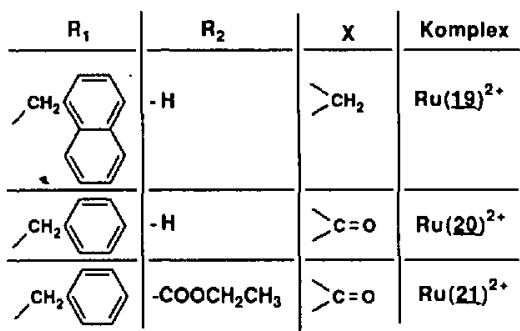

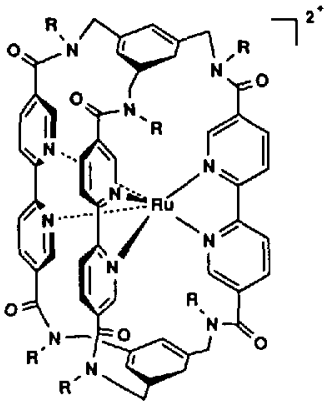

$\mathrm{P}=, \mathrm{CH}_{2} \longrightarrow$

$\operatorname{Ru}(\mathbf{2 2})^{2+}$ losen Desaktivierungsprozesse (s. Fig.5) an Bedeutung gewinnen.

Als neue Strategie wurde versucht, die drei bpy-Einheiten oktaedrisch um das Metall-Zentrum anzuordnen und sie gleichzeitig in einer Käfigstruktur zu fixieren. Die bis heute hergestellten Halbkäfigund Käfig-Komplexe sind unten zusammengestellt.

Synthetisch bietet besonders die Verbindung $[\mathrm{Ru}(\mathbf{2 2})]^{2+}$ einigen Anreiz. Da der übliche Syntheseweg über den Einbau des $\mathrm{Ru}(\mathrm{II})-\mathrm{Kations}$ in den freien Liganden [21] aus kinetischen Gründen (ausschliessliche Produktion von Polymermaterial) nicht beschritten werden konnte, wurde mittels einer Templat-Reaktion der Käfig-Ligand um das Metall-Zentrum aufgebaut. Die entsprechende Reaktionsabfolge ist dem Schema zu entnehmen. Beschränkend auf eine weitere Anwendbarkeit dieser Synthese ist die im letzten Synthese-Schritt auftretende Möglichkeit der Bildung von offenkettigen, polymeren, mehrkernigen Ru-Komplexen. Das Arbeiten in grosser Verdünnung wirkt dieser Nebenreaktion entgegen. Ausserdem reagieren beim gleichen Syntheseschritt 12 funktionelle Gruppen (6 Säurechlorid- und 6 sekundäre Amin-Funktionen) simultan miteinander. Aus diesem Grund sind ausserordentlich reine Edukte für diesen Schritt erforderlich. Dic bis heute erreichte geringe Ausbeute an $[\mathrm{Ru}(22)]^{2+}$ liegt zwischen $1-5 \%$ und ist mit den obgenannten Gründen erklärbar.

In Fig. 15 sind die wichtigsten Eigenschaften von $[\mathrm{Ru}(22)]^{2+}$ dargestellt.

Im Vergleich zu $\left[\mathrm{Ru}(\mathrm{bpy})_{3}\right]^{2+}$ hat der Käfig-Komplex dieselben Absorptionseigenschaften mit Ausnahme des etwas kleineren $\varepsilon$-Wertes (s. Fig. 16).

Die Lebensdauer des angeregten $\mathrm{Zu}$ standes bei Raumtemperatur ist deutlich länger (Fig. 10), was mit der starren Struktur des Komplexes erklärt werden kann [22]. Eine lange Lebensdauer des angeregten Zustandes wirkt sich hingegen günstig auf die Eigenschaften aus, welche ein Pho- tosensibilisator aufweisen sollte. Aus den elektrochemischen Daten der Käfig-Verbindung ist zu schliessen, dass sie im ange- regten Zustand ein stärkeres Oxidationsmittel (Fig. 2 und 3) als die entsprechende Verbindung $\left[{ }^{*} \mathrm{Ru}(\mathrm{bpy})_{3}\right]^{2+}$ darstellt.

Die markanteste Anderung ist im photochemischen Verhalten zu beobachten. Die Verbindung zeigt, wie erwartet, keinerlei Reaktivität bezüglich der Photosubstitution. Die gemessene Steigerung der Photostabilität liegt in der Grössenordnung $>10^{4}$.

Da der Faktor der Quantenausbeuten $\Phi_{\text {Rac }} / \Phi_{\text {Sub }}$ beim Komplex [Ru(bpy) $\left.)_{3}\right]^{2+}$ etwa 100 beträgt, bildet die Messung der Photoracemisierung eine empfindliche Methode, um die photochemische Aktivität unabhängig von der Messung der Photosubstitution zu bestimmen. Es gelang uns aber nicht, $[\operatorname{Ru}(22)]^{2+}$ auch nur teilweise in ihre Enantiomeren zu zerlegen. Es wurde daher der Halbkäfig-Komplex $[\mathrm{Ru}(20)]^{2+}$ in die Untersuchung miteinbezogen. Auch bei $[\mathrm{Ru}(\mathbf{2 0})]^{2+}$ konnte weder eine Reaktivität bezüglich der Photosubstitution noch eine Photoracemisierung beobachtet werden. Es genügt offensichtlich, die Liganden einseitig starr zu verbinden, um jede Photore-

\section{Schema}
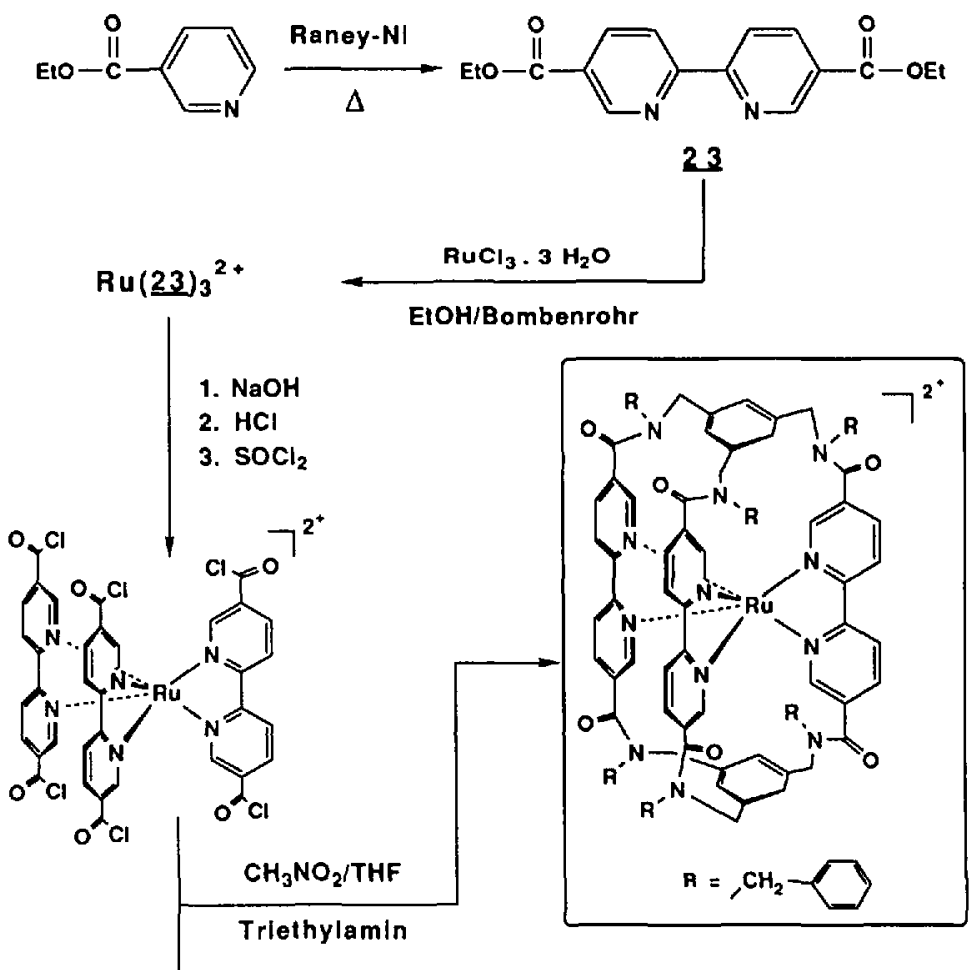

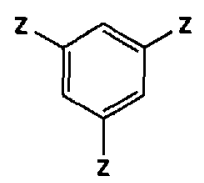

$\mathrm{Z}=-\mathrm{CH}_{2}-\mathrm{NH} \cdot \mathrm{CH}_{2} \longrightarrow$
$\mathrm{BH}_{3} \cdot \mathrm{THF}$<smiles>[Y]c1cc([Y])cc([Y])c1</smiles>

$Y=$<smiles>CC(=O)NCc1ccccc1</smiles><smiles>[X]c1cc([X])cc([X])c1</smiles>

Toluol

Benzylamin/Triethylamin

$x=-c-c r$ 
aktion zu verhindern. Die entscheidenden Faktoren, welche die photochemische Reaktivität beeinflussen, sind die Starrheit und die 'Passform' des Käfig-Gerüstes. So reagieren die beiden Verbindungen $[\operatorname{Ru}(18)]^{2+}$ und $[\mathrm{Ru}(19)]^{2+}$ wegen ihrer flexibleren Käfig-Struktur bei der Einstrahlung in ihre ${ }^{3}$ MLCT-Bande via Photosubstitution und Photoracemisierung.

Es konnte gezeigt werden, dass bei geeigneter Änderung der Ligand-Struktur - drei bpy-Einheiten $\rightarrow$ Käfig-Struktur - gewisse Nachteile der Standardverbindung behoben oder sogar in einen Vorteil umgewandelt werden können. Zukünftige Arbeiten in diesem Gebiet konzentrieren sich auf einen Syntheseweg, welcher höhere Ausbeuten und 'funktionalisierte Käfig-Komplexe' liefert, die zum Aufbau supramolekularer Strukturen geeignet sind.

\section{Polynukleare Ru-Komplexe}

Die Erforschung mehrkerniger $\mathrm{Ru}(\mathrm{II})$ diimin-Komplexe bildet ein Tätigkeitsfeld, das auf den gesamten Erfahrungen der mononuklearen $\mathrm{Ru}$-Verbindungen aufgebaut ist und gleichzeitig als Vorstufe der 'supramolekularen' Komplex-Chemie angesehen werden kann. Eine wichtige Fragestellung in diesem Gebiet lautet: Welche Distanz ist zwischen zwei durch einen Brückenliganden getrennte Metall-Zentren notwendig, damit sich die beiden Zentren nicht mehr beeinflussen? Diese Problematik entspricht genau den Verhältnissen, wie sie bei einem Ladungs- bzw. Energietransfer auftreten, bei dem z. B. ein Elektron sich über eine gewisse Distanz bewegt und anschliessend genügend Zeit hat, sich an einer chemischen Reaktion zu beteiligen (Ladungsseparation; Photosynthese) [23]. Um diese Frage zu beantworten, müssen drei Randbedingungen festgelegt werden:

a) Nach der Absorption eines Photons muss das 'Antwortsignal' eindeutig aus demjenigen Metall-Zentrum kommen, welches anfänglich nicht am Absorptionsprozess beteiligt war. Diese Bedingung kann durch unterschiedliche Metall-Zentren oder gleiche Metallzentren mit unterschiedlichen Ligand-Sphären erfüllt werden.

b) Der Brückenligand muss eine räumlich starre Anordnung einnehmen, damit die Metall-Zentren auf einer wohldefinierten 'Distanz' gehalten werden.

c) Die Experimente müssen in verdünnten Lösungen durchgeführt werden, um die Möglichkeit intermolekularer Prozesse zu verringern.

In Fig. 17 ist eine Anordnung gezeigt, welche den besprochenen Bedingungen entsprechen würde.

Der Photosensibilisator $M_{1}$ gelangt nach der Einstrahlung eines Photons durch einen MLCT-Übergang in einen angeregten Zustand, d.h. ein Elektron wird in den Brückenligand promoviert. Der Donor-Li-

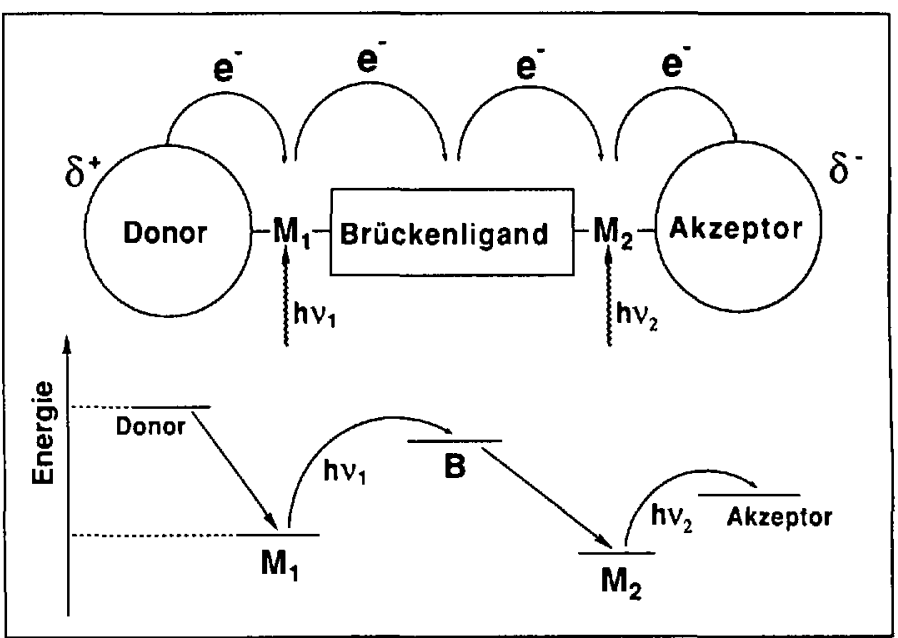

Fig. 17. Aufbau eines binuklearen Metall-Komplexes, bei dem eine Lichtabsorption eine Ladungsseparation bewirken kann

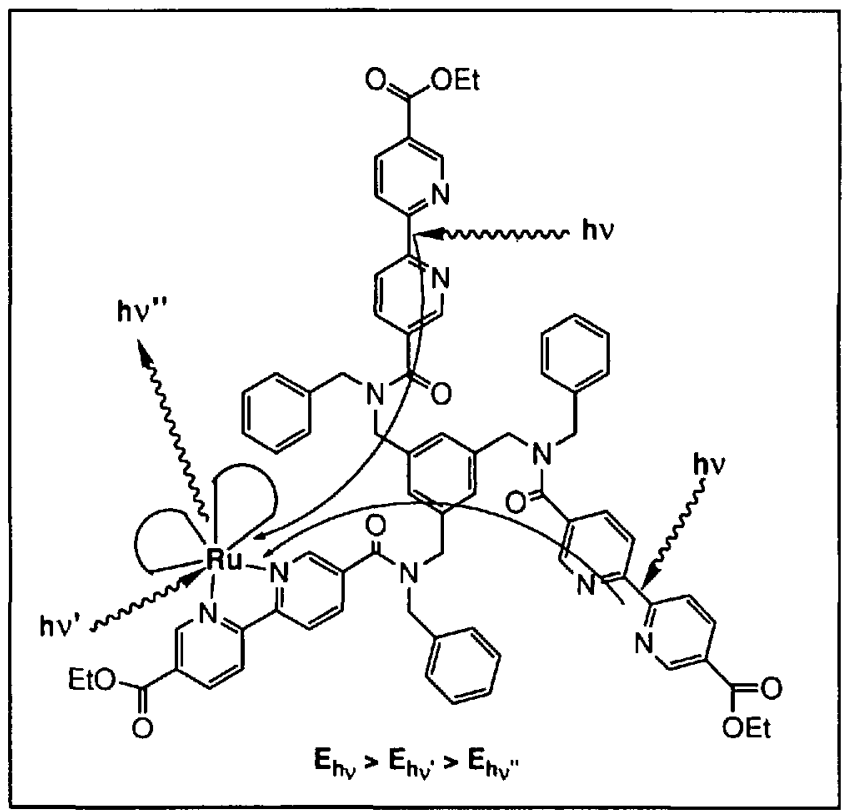

Fig. 18. Beispiel eines intramolekularen Energietransfers [24]

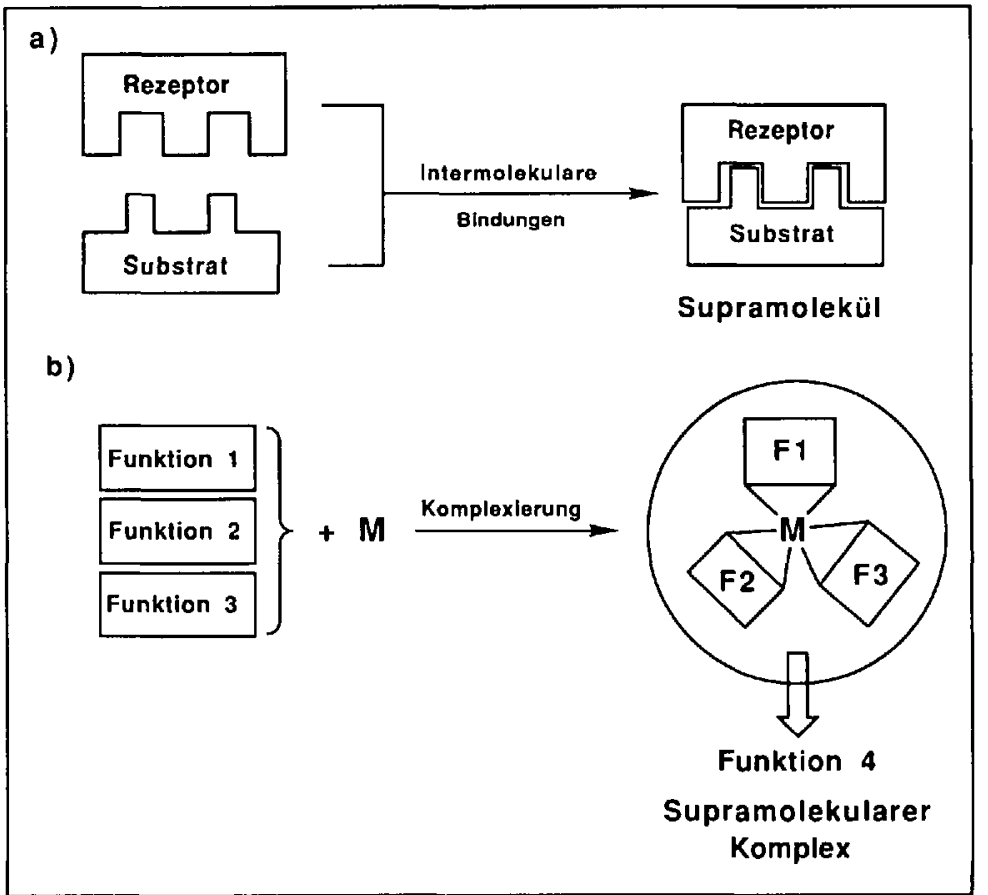

Fig. 19. a) Supramolekül, welches auf dem 'Erkennungsprinzip' aufgebaut ist. b) Supramolekularer Komplex, bei dem die Funktionen 1,2 und 3 eine 'neue' Funktion 4 aufbauen 
gand reduziert in einem anschliessenden Schritt das oxidierte Metall-Zentrum $\mathbf{M}_{1}$. In einem konzertierten Redox-Prozess übernimmt der Akzeptor-Ligand ein Elektron vom Metall-Zentrum $\mathrm{M}_{2}$ und der negativ geladene Brückenligand reduziert das oxidierte Metall-Zentrum $\mathbf{M}_{2}$. Die Nettoreaktion kann als Verschiebung eines Elektrons vom Donor- auf den Akzeptor-Liganden beschrieben werden. Das auf dem Akzeptor-Ligand lokalisierte Elektron kann dann, wie oben beschrieben wurde, in einer weiteren Reduktionsreaktion eingesetzt werden.

Es ist leicht einzusehen, dass beim Aufbau solcher polynuklearer Metall-Komplexe grosse Variationsmöglichkeiten bestehen. So können z. B. bei einer binuklearen Verbindung sowohl der Donor-, Akzeptor- und der Brückenligand als auch das Metall-Zentrum $M_{1}$ und $M_{2}$ variiert werden. Die energetische Lage der Orbitale der beteiligten Molekülbausteine müssen so abgestimmt sein, dass die Elektronen nur den Weg vom Donor-zum AkzeptorLigand beschreiten können (Fig. 17).

Erste Resultate konnten mit dem Brükkenliganden 21 gewonnen werden. Die Halbkäfig-Liganden 18-21 eignen sich in ausgestreckter, ebener Form ausgezeichnet als polynukleare Brückenliganden (s. Fig. 18).

Die Verbindung $\left[R u(b p y)_{2}(21)\right]^{2+}$ zeigt keinerlei Fluoresenz aus den beiden nicht komplexierten bpy-Einheiten, die beim freien Liganden 21 bei $77 \mathrm{~K}$ beobachtet werden kann. Es findet also ein effizienter, intramolekularer Energietransfer zwischen den nicht koordinierten bpy-Einheiten und dem mit dem Ru-Metall komplexierten Teil des Liganden statt [24].

\section{8. 'Supramolekulare' Komplexe}

Es würde den Rahmen dieses Artikels sprengen, den aktuellen Stand der Forschung auf dem Gebiet der 'supramolekularen Chemie' und im speziellen der supramolekularen Komplexe darzustellen. Eine einheitliche Definition dieses Begriffs existiert nicht. Es muss daher das Auftreten und die Wirkungsweise der supramolekularen Komplexe auf beschreibende Art erklärt werden.

Supramoleküle zeichnen sich durch einen höheren Grad an 'Organisation' aus, als dies bei einfachen Molekülen der Fall ist. Die 'Organisation' beruht auf schwachen intermolekularen Bindungen (z.B. van der Waals), welche zwischen den verschiedenen Molekülen, Ionen oder Atomen aufgebaut wird. Der Ordnungsgrad bei Supramolekülen ist grösser, es finden Wechselwirkungen zwischen den Untereinheiten statt und gleichzeitig treten neue, charakteristische Funktionen oder Eigenschaften auf [25]. Fig. 19 zeigt den Aufbau supramolekularer Aggregate oder Komplexe.

In $a$ ) ist ein Beispiel aus der Biochemie dargestellt, bei dem ein Rezeptor-Molekül ein entsprechendes Substrat erkennt und einen grösseren Verband bildet, welcher wiederum eine neue Funktion ausüben kann. Ein von $a$ ) etwas abweichendes Konzept ist in $b$ ) gezeigt. Liganden mit ausgesuchten, unterschiedlichen Funktionen werden an ein Metall-Zentrum gebunden und bilden ein Molekül, welches eine neue, globale Funktion besitzt, die die einzelnen, komplexierten Liganden nicht aufweisen [26]. Es sind nicht die intermolekularen Wechselwirkungen, die der Komplex-Verbindung die Eigenschaften eines Supramoleküls verleihen, sondern das $\mathrm{Zu}$ sammenwirken der Einzelfunktionen zu einer übergeordneten 'höheren' Funktion. Schon Aristoteles hat in seinem Zitat 'Das Ganze ist mehr als die Summe der Teile' (Originaltext: to y $\alpha \rho$ odov $\pi \rho o t \varepsilon \rho o v$

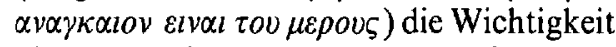
dieses Prinzips erkannt. Der Aufbau solcher funktionalisierter Supramoleküle ist von grösstem Interesse, da bei geeigneter Wahl der Bausteine natürliche Prozesse (z.B. die Photosynthese) simuliert oder Reaktionsabläufe (z.B. Katalyse) unterstützt werden können.

$\mathrm{Ru}(\mathrm{II})$-diimin-Komplexe bilden aus verschiedenen Gründen ideale Bausteine, um supramolekulare Verbindungen aufzubauen. Die Herstellung und die Eigenschaften der mononuklearen Ru-Komplexe sind eingehend untersucht worden. Die Verbindungen zeigen sich sehr empfindlich (Absorption, Emission, Elektrochemie) gegenüber Änderungen, welche in der Ligand-Sphäre vorgenommen werden. Ausserdem können sie leicht zu grösseren Aggregaten - polynukleare Komplexe zusammengefügt werden. Ein Beispiel soll den Aufbau einer solchen neuen Funktion illustrieren.

In Fig. 20 ist der trinukleare Komplex $\left[\left(\operatorname{Ru}(\mathrm{bpy})_{2}\right)_{2}\left(\mathrm{Os}(\mathrm{bpy})_{2}\right)(\mathbf{2 0})\right]^{6+}$ abgebildet. Er besteht aus zwei Bausteinen $\left[\mathrm{Ru}(\mathrm{bpy})_{2}(\mathbf{2 0})\right]^{2+}$ und einer $\left[\mathrm{Os}(\mathrm{bpy})_{2}(\mathbf{2 0})\right]^{2+}$-Einheit, welche alle als ge- trennte Komplexe Licht absorbieren und eine starke Emission aufweisen. Die Emission von $\left[\mathrm{Os}(\mathrm{bpy})_{2}(\mathbf{2 0})\right]^{2+}$ liegt energetisch etwas tiefer und ist daher von derjenigen der Einheit $\left[\mathrm{Ru}(\mathrm{bpy})_{2}(\mathbf{2 0})\right]^{2+}$ unterscheidbar. Erste Resultate zeigen, dass im trinuklearen Komplex die Emission aus dem 'Osmium-Arm' kommt [27]. Die Emission aus den Ru-Komplexen wird vollständig gelöscht. Es findet ein Energietransfer vom Ru auf das Os statt. Dieser Effekt kann treffend mit dem Begriff einer 'EnergieSammelantenne' [28] für die Os-Einheit umschrieben werden. Diese neue Eigenschaft der Energiekollektion des trinuklearen Komplexes ist in den mononuklearen Komplexen als Funktion nicht vorhanden.

Weitere kooperative Effekte können durch Variation der Einzelfunktionen konstruiert werden. Der Aufbau und die Erforschung der Chemie der supramolekularen Ru-Komplexe hat erst begonnen.

\section{Ausblick}

Der vorliegende Artikel versucht, deutlich zu zeigen, dass der Wissensstand bei der Herstellung und der Interpretation der Eigenschaften von mononuklearen Ru(II)diimin-Komplexen weit fortgeschritten ist, und daher die Zahl der Forschungsarbeiten, welche in diesem Gebiet veröffentlicht werden, eher abnimmt. Umgekehrt wird das Interesse in Zukunft vermehrt auf der Konstruktion und Untersuchung polynuklearer und im besonderen supramolekularer Ru-Komplexe liegen. Im Bereich der möglichen technischen Anwendung solcher Ru-Verbindungen können die Arbeiten von Grätzel [29] erwähnt werden. Bei der Umwandlung von Sonnenenergie in elektrische Energie mittels einer photovoltaischen Zelle spielen Ru(II)-diimin-Komplexe, die an einer 'fraktalen' $\mathrm{TiO}_{2}-\mathrm{Mem}$ bran absorbiert sind, die entscheidende Rolle als Photosensibilisatoren.

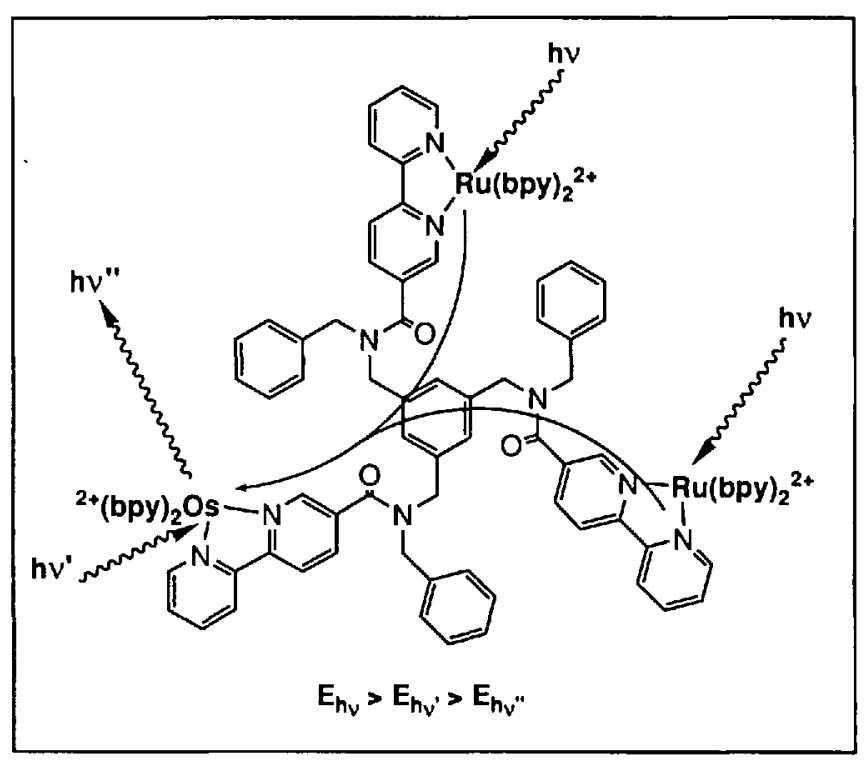

Fig. 20. Energiesammel- oder Antenneneffekt. Es kann nur die Emission aus dem 'OsKomplex' beobachtet werden [27]. 
Der Autor dankt dem Schweizerischen Nationalfonds zur Förderung der Wissenschaftlichen Forschung und der Universitü̈t Fribourg für die finanzielle Unterstützung und den Kollegen aus der 3-Zentren-Forschungsgruppe Fribourg/Bologna/Bonn gilt ein spezieller Dank für die Anregungen und intensiven Diskussionen.

Eingegangen am 23. Januar 1990

[1] K. Kalyanasundaram, Coord. Chem. Rev. 1982, 46.159

[2] a) F. Barigelletti, A. Juris, V. Balzani, P. Belser, A. von Zelewsky, Inorg. Chem. 1983, 22, 3335 b) P. Belser, A. von Zelewsky, A. Juris, F. Barigelletti, V. Balzani, Gazz. Chim. Ital. 1983, 1/3, 731 ; c) P. Belser, A. von Zelewsky, A. Juris, F Barigelletti, V. Balzani, Chem. Phys, Letr. 1984 104, 100; d) V. Balzani, A. Juris, F. Barigelletti, P. Belser, A. von Zelewsky, Riken Q. 1984, 78, 78; e) T. J. Meyer, Acc. Chem. Res. 1989, 22, 163 f) E. A. Seddon, K.R. Seddon, 'The Chemistry of Ruthenium', Elsevier, Amsterdam, 1984, Kap. 15.

[3] a) A. Juris, V. Balzani, F. Barigelletti, S. Campagna, P. Belser, A. von Zelewsky, Coord. Chem. Rev. 1988, 84, 85; b) M. Kirch, J. M. Lehn, J.P. Sauvage, Hels. Chim. Acta 1979, 62, 1345.

[4] A. von Zelewsky, G. Gremaud, Helv. Chim. Acta 1988, 71,1108

[5] a) W. Kaim, S. Ernst, St. Kohlmann, Chem. in unserer Zeit 1987, 2l, 50; b) D. Beer, Diplomarbeit, Universität Fribourg, 1980.

[6] a) P. Belser, A. von Zelewsky, Helv. Chim. Acta $1980,63,1675$; b) A. Juris, V. Balzani, P. Belser, A. von Zelewsky, ibid. 1981, 64, 2175; c) A. Juris, $\therefore$. Barrigelletti, V. Balzani, P. Belser, A. von Zelewsky, /sr.J. Chem. 1982, 22, 87; d) M. Maestri, D. Sandrini, V. Balzani, P. Belser, A. von Zelewsky, Chem. Phys. Lett 1984, 110, 611; e) P Belser, A. von Zelewsky, A. Juris, F. Barigelletti. V. Balzani. Gazz. Chim. Ital. 1985, ll5, 723; f) D. Sandrini, M. Maestri, P. Belser, A. von Zelewsky, V. Balzani J. Phys. Chem. 1985, 89, 3675; g) F Barigelletti, P. Belser, A. von Zelewsky, A. Juris
V. Balzani, ibid. $1985,89,3680$; h) A. Juris, F Barigelletti, V. Balzani, P. Belser, A. von Zelewsky, J. Chem. Soc., Faraday Trans. 2 1987, 83 2295.

[7] a) P. Belser, A. von Zelewsky, M. Zehnder, Inorg. Chem. 1981, 20, 3098; b) St. Kohlmann, S. Ernst, W. Kaim, Angew. Chem. 1985, 97, 698; c) K Haarmann, Diplomarbeit, Universität Fribourg, 1986.

[8] a) R. Krause, Structure Bonding 1987, 67, 1; b) E. C. Constable, P. J. Steel, Coord. Chem. Rev $1989,93,205$

[9] R. Gillard, R. Hill, J. Chem. Soc., Dalton Trans. 1974, 1217.

[10] a) P. Belser, L. De Cola, A. von Zelewsky, $J$ Chem. Soc., Chem. Commun. 1988, 1057; b) L. De Cola, F. Barigelletti, V. Balzani, P. Belser, A. von Zelewsky, F. Vögtle, F. Ebmeyer, S. Grammenudi, $J$. Am. Chem. Soc. 1988, 110, 7210; c) F. Barigelletti, L. De Cola, V. Balzani, P. Belser, A von Zelewsky, F. Vögtle, F. Ebmeyer, S. Grammenudi, ibid. 1989, 111,4662 ; d) Ch.O. Dietrich Buchecker, J.P. Sauvage, Chem. Rev. 1987, 87, 795 .

111 a) A Juris, P. Belser, F. Barigelletti, A von Zelewsky, V. Balzani, Inorg. Chem. 1986, 25, 256; b) F. Barigelletti, A. Juris, V. Balzani, P. Belser, A. von Zelewsky, ibid. 1987, 26, 4115; c) F. Barigelletti, A. Juris, V. Balzani, P. Belser, A. von Zelewsky, in 'Photochemistry and Photophysics of Coordination Compounds', Ed. H. Yersin und A. Vogler, Springer-Verlag, Berlin-Heidelberg, 1987 , p. 79 .

[12] a) P. Belser, C. Daul, A. von Zelewsky, Chem. Phys. Lett. 1981, 79, 596; b) C.M. Elliott, E. J. Hershenhart, J. Am. Chem. Soc. 1982, 104, 7519

[13] a) P. Belser, A. von Zelewsky, A. Juris, F. Barigelletti, A. Tucci, V. Balzani, Chem. Phys. Lett. 1982. 89,101 ; b) A. Juris, F. Barigelletti, V. Balzani, P. Belser, A. von Zelewsky, Inorg. Chem. 1985, 24 , 202 ; c) F. Barigelletti, A. Juris, V. Balzani, P. Belser, A. von Zelewsky, J. Phys. Chem. 1987, 91, 1095.

[14] P. Belser, Dissertation Nr. 805, Universität Fribourg, 1979
[15] F. Barigelletti, A. Juris, V. Balzani, P. Belser, A von Zelewsky, J. Phys. Chem. 1986, 90, 5190.

[16] a) G. Blaschke, Angew'. Chem. 1980, 92, 14; b) D. Kotkar, P. K. Ghosh, Inorg. Chem. 1987, 26, 208; c) B. E. Buchanan, E. McGovern, P. Harkin, J.G. Vos, Inorg. Chim. Acsa 1988, 154, 1.

[17] a) U. von Arx, Diplomarbeit, Universität Fribourg, 1980; b) G. Liebich, Diplomarbeit, Universität Fribourg, 1980; c) W. Luginbühl, Diplomarbeit, Universität Fribourg, 1985.

[18] a) G. Malouf, P.C. Ford, J. Am. Chem. Soc. 1974, 96, 601; b) G. Malouf, P.C. Ford, ibid. 1977, 99, 7213; c) J. van Houten, R.J. Watts, Inorg. Chem. 1978, 17,3381.

[19] J. Schöpfer, Diplomarbeit, Universität Fribourg. 1989.

[20] B. Durham, S. R. Wilson, D.J. Hodgson, T.J. Meycr, J. Am. Chem. Soc. 1980, 102, 601

[21] a) S. Grammenudi, F. Vögtle, Angew: Chem. Int. Ed. 1986, 25, 1122; b) S. Grammenudi, M. Franke, F, Vögtle, E. Steckhan, J. Incl. Phenom. $1987,5,695$.

[22] Die ideale 'Passform' des Käfigs konnte durch ein CPK-Modell ermittelt werden.

[23] a) R.A. Marcus, N. Sutin, Biochim. Biophys. Acta $1985,811,265$; b) B. Kräutler, Chimia 1987, 4l, 277.

[24] L. De Cola, P. Belser, F. Ebmeyer, F. Barigelletti, F. Vögtle, A. von Zelewsky, V. Balzani, Inorg. Chem., zur Publikation angenommen.

[25] J. M. Lehn, Angew. Chem. 1988, 100,91.

26] J.M. Lehn, in 'Supramolecular Photochemistry', Ed. V. Balzani, D. Reidel Publishing Company, Dordrecht, 1987, S. 29.

[27] V. Balzani, P. Belser, L. De Cola, A. von Zelewsky, unveröffentliche Resultate.

[28] V. Balzani, L. Moggi F. Scandola, in 'Supramolecular Photochemistry', Ed. V. Balzani, D. Reidel Publishing Company, Dordrecht, 1987, S.1.

[29] a) M. Grätzel, Acc. Chemt. Res. 1981, 14, 376; b) P. Belser, B. Jenny, L. Walder, Konferenzbericht des EPA-Workshops, Adelboden 2.-4. Oktober 1989, EPA Newsletter, zur Publikation angenommen.

\section{Applications of Inductively Coupled Plasma Mass Spectrometry (ICP-MS) in the Central Analytical Department of Ciba-Geigy ${ }^{*}$}

\section{Heinrich Baumann**}

Ahstract. The present paper shows the potentialities of ICP-MS for the trace and ultratrace analysis of different materials in an industrial laboratory. An overview is given on some applications of ICP-MS in the Central Analytical Department of Ciba-Geigy. The use of a He-MIP (Microwave Induced Plasma) as an alternative to the Ar-ICP as an ionization source for the mass spectrometer is discussed.
One application shown is the determination of traces of Pt-group metals in pharmaceuticals and agrochemicals. $\mathrm{Ru}, \mathrm{Rh}$, $\mathrm{Pd}$, and $\mathrm{Pt}$ are used as hydrogenating catalysts. Catalyst losses have to be controlled because of economical and ecological reasons. After digesting the samples with $\mathrm{HNO}_{3} / \mathrm{HCl}$ in a Carius tube, detection limits in the lower $\mathrm{ng} / \mathrm{g}$ range can be reached for those metals.

Another application is concerned with the determination of toxic heavy metals in iron oxides. Those iron oxides are the by-

\footnotetext{
* Based on a lecture presented at the 10th meeting of the 'Groupe d'Analyse Chimique Interdisciplinaire de l'EPFL, GACHI: ICP-MS' on November 28, 1990 in Lausanne. For introductory remarks, see Chimia 1990. 44,92 .
}

** Correspondence Dr. H. Baumann

Central Analytical Lespartment

Ciba-Geigy Ltd., R-1055.4.02

$\mathrm{CH} 4002$ Basel 